\title{
Plasma Bioscience and Medicines
}

\author{
Received 18 May, 2021; revised 9 June, 2021; accepted 23 June, 2021
}

\author{
Eun Ha Choi*, Young June Hong, and Nagendra Kumar Kaushik \\ Department of Electrical and Biological Physics, Plasma Bioscience Research Center \& Applied Plasma Medicine \\ Center, Kwangwoon University, Seoul 01897, Republic of Korea
}

\section{*Corresponding author E-mail: ehchoi@kw.ac.kr}

\begin{abstract}
The nonthermal atmospheric biocompatible plasma, also called cold plasma, is the fourth state of matter, is a partially ionized gas consisting cocktail of gas's molecules, free radicals, ions, electrons, and physical components such as photons, electric field and some heat.

It has been successfully used in the biomedical, agricultural food safety, environment applications including industrial application for the processing of materials and etc. for not mentioned here. The recent discovery of its efficacy in sterilization of microorganisms has trigged a large quantity of research in the biomedical field. Here we review configurations and electrode layouts of typical plasma device for applications to biomedical such as cancer treatment and virus inactivation technology. Cocktail of reactive oxygen and nitrogen species (RONS) would be efficient and effective to several biomedical applications such as inactivation of drug-resistant bacteria, cancer treatment, inactivation of viruses, skin and dental applications. This detailed review provides an outline of typical plasma sources, their physical and chemical (RONS) characteristics based on their diagnostic methods, including cancer treatment strategies and inactivation of viruses. This review also emphasizing on strategises to control and inactivation of SARS-COV2 (COVID19) and rejuvenate lung cells. Plasma bioscience and medicine technologies will deliver a new model of therapeutic clinical systems along with sustainable application to environmental issues.
\end{abstract}

Keywords: Nonthermal atmospheric pressure biocompatible dielectric barrier discharged plasma, Cold plasma, Virus inactivation, Cancer treatment, Plasma bioscience and medicine

\section{Introduction}

Plasma is a group of electrically charged gases also called the fourth state of matter or ionized gas after the solid, liquid, and gaseous states. This material was created about 13.7 billion years ago, and is the basic and fundamental material for universe creation, and to understand the meaning of plasma, we can find it by looking at the Sanskrit word, plasma. "Pla or pra" means very basic, primitive, very high, "z or s" means life, water, and "ma" means a matter with energy $[1,2]$. When it is combined to interpret the meaning, it is an essential substance or material in which the energy is accumulated. Or it can be interpreted as fundamental and life-forming quantum matter. In medicine, because "blood" was viewed as the fundamental and essential material of life, "plasma" was called plasma. In biology, it was called plasma because the "proplasm" of cells was viewed as the basic material of life [3-5].

Plasma could be generated through the electric discharge from the gas between the powered and grounded electrodes with direct current (DC) or alternating current (AC) voltage [6-12]. The electric discharges produce electrons, ions, light, heat, and reactive neutral gases, which are in high energy excited states, along with the neutral gases in ground state energy [12-15]. Interactions of the plasma generated reactive oxygen species and nitrogen species (RONS) with biological cells or tissues could be used for wound treatment, selective cancer apoptosis, and microbial biofilm eradication. Reactive gases with high excited energy are represented by an oxidative potential, which refers to the ability to take electrons from surrounding molecules or atoms. At this time, the atoms or molecules from which the electrons were deprived are called to be oxidized. Among the reactive species, the reactive oxygen species (ROS) are hydroxyl groups $\mathrm{OH}$ with the oxidative potential of $2.8 \mathrm{eV}$, oxygen atoms $\mathrm{O}$ with oxidative potential of
$2.4 \mathrm{eV}$, ozone $\mathrm{O}_{3}$ with oxidative potential of $2.1 \mathrm{eV}$, hydrogen peroxide $\mathrm{H}_{2} \mathrm{O}_{2}$ with oxidative potential of $1.8 \mathrm{eV}$, and oxygen molecules $\mathrm{O}_{2}$ with oxidative potential of $1.2 \mathrm{eV}$, superoxide anion $\mathrm{O}_{2}{ }^{*-}$ with an oxidative potential of $\sim 1.3 \mathrm{eV}$, and superoxide di-anion $\mathrm{O}_{2}{ }^{*--}$ with oxidative potential of $\sim 1.6 \mathrm{eV}$, while the reactive nitrogen species (RNS) are nitrogen oxides $\mathrm{NO}$ with oxidative potential of $\sim 1.7 \mathrm{eV}$, nitrite $\mathrm{NO}_{2}{ }^{-}$, nitrite di-anion $\mathrm{NO}_{2}{ }^{--}$, and peroxynitrite $\mathrm{ONOO}^{-}$[16-18]. It also contains electromagnetic waves composed of a combination of ultraviolet, visible, and infrared emissions from discharge gases such as helium (He), argon (Ar), nitrogen $\left(\mathrm{N}_{2}\right)$, air, and their mixtures and compounds. Low-temperature plasma generated at atmospheric pressure generally maintains the form of a cocktail in which these active gases are mixed. In particular, this type of low-temperature plasma applicable to a living body is simply called nonthermal atmospheric pressure biocompatible dielectric barrier discharged plasma (NBP). In other words, it is also referred to as nonthermal atmospheric pressure plasma (NAP) $[13-15,19-24]$ and cold atmospheric pressure plasma (CAP) [25-40]. Here, we may call it NBP. This kind of NBP has a slight thermal effect, as well as electrons and ions, reactive oxygen and nitrogen gases, ultraviolet and visible light for applications to plasma medicine [41-47], agriculture [48-59], antibacterial treatment against dental disease, disc herniation treatment and antifungal treatment [60-70].

The purpose of this review would provide a general survey and recent results of plasma bioscience and medicines for applications mainly to healthcare and hygiene, which is based on the convergence of plasma physics, chemistry, biology, and medicines. Current studies of plasma biosciences and medicine are reviewed, with an emphasis on NBP sources, their plasma diagnostics, and main applications to plasma medicines such as cancer treatment and microbial treatment of bacteria removal and virus inactivation. This review covers typical NBP 
sources which would be widely used in the world, and their basic characteristics will be briefly explained. Most NBP plasma sources are belonging to dielectric barrier discharged plasmas, in which pencil-type jets and surface discharged or facing discharged plasma are frequently used among the many plasma sources with their driving frequencies are low less than $1 \mathrm{kHz}$, medium ranging from $\sim 10-100 \mathrm{kHz}$, and high ranging from $\sim \mathrm{MHz}$ up to $\mathrm{GHz}$. These NBP plasmas may be characterized by specified categories since their electron density is in between $10^{12}$ to $\sim 10^{16} \mathrm{~cm}^{-3}$, which is somewhat different from glow and arc discharge plasmas. However, their electron temperatures are in the range of $0.8-3 \mathrm{eV}$, which is quite similar to normal glow plasmas. The plasma gas temperature in plasma plumes would be very important for patients since the heat above $45^{\circ} \mathrm{C}$ should not be allowed by regulation. Also, the harmful gases, particularly ozone $\mathrm{O}_{3}$, must be kept less than $0.05 \mathrm{ppm}$ during $8 \mathrm{~h}$ working conditions in $8 \mathrm{~m}^{3}$ space volume.

The fundamental issues occurring from these NBP interactions with water or biological materials are the generation of ROS as well as RNS. Figure 1 demonstrates the schematic of a basic mechanism that shows how RONS, such as $\mathrm{O}_{3}, \mathrm{OH} \cdot \mathrm{H}_{2} \mathrm{O}_{2}, \mathrm{NO}, \mathrm{NO}_{2}$, and ultraviolet (UV), which are coexisting in NBP, may be generated, and delivered to water or biological tissues during plasma interactions, even though plasma electrons and ions cannot propagate directly into water or skin layers. Here, we also establish the fast method of plasma-initiated UV photolysis and the slow diffusion processes for RONS to penetrate water or tissue for plasma biosciences and medicine. Their synergistic interactions could augment RONS penetration into cells or water in the biological tissue for health care and medicine.

Figures 1(a) and 1(b) show atopy, skin cancer, and other skin lesions or their internal tissues could be treated to get healing effects throughout RONS penetration and their interactions with pathogenic tissue areas [41-48]. In modern medicine, incurable diseases such as modern skin diseases, dementia, and cancer occur due to the mutational transformation of normal cells and related cells. In addition, incurable diseases like Alzheimer's and Parkinson's disease, which are related to protein variables, can be fundamentally treated using a RONS composed of simple molecules, that is, plasma, which is the fundamental material of life. For this, the next generation plasma medicine could be pioneered. It can also be used from the viewpoint of oral health, oral dentistry, and skin care. In addition, it can be used for life sciences such as cancer treatment, agricultural and fishery food processing and storage, environment, and health cares through the interaction of the dissolved RONS in plasma-activated water (PAW) made by treating plasma onto water, with cell, bacteria, and fungi [71-78].

\section{Nonthermal atmospheric pressure biocompat- ible dielectric barrier discharged plasma (NBP) generator}

Plasma devices generated at atmospheric pressure must maintain the concentration of harmful gases such as ozone less than $0.05 \mathrm{ppm}$ in the living environment $[79,80]$, and there must be no electric shock, and heat to living tissues to be applied to bioscience and medical science. In other words, it should be put into real-life and medical health sites to ensure electrical safety and biomedical safety, so that it is safe and convenient for all people. There are two representatives of NBP, so-called "soft plasma jet", as shown in the first left at the top of Fig. 2 , and surface discharged micro dielectric barrier discharge plasma, so-called "surface NBP", in the first and the second left at the bottom of Fig. 2 [4]. The NBP, which is used all over the world, has a slightly modified form of these two or a combination of these two types for face-to-face discharge. "Plasma jet", which is relatively easy to manufacture worldwide, is currently the most widely used, and surface NBP whose electrode is based on the patent from plasma display panel technology, could be used by PBRC (Plasma Bioscience Research Center) to lead the development of health medicine, environment, agriculture

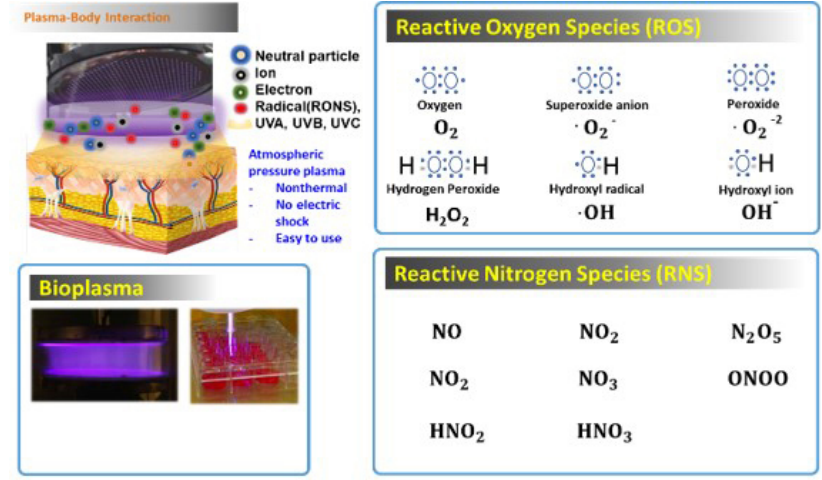

(a)

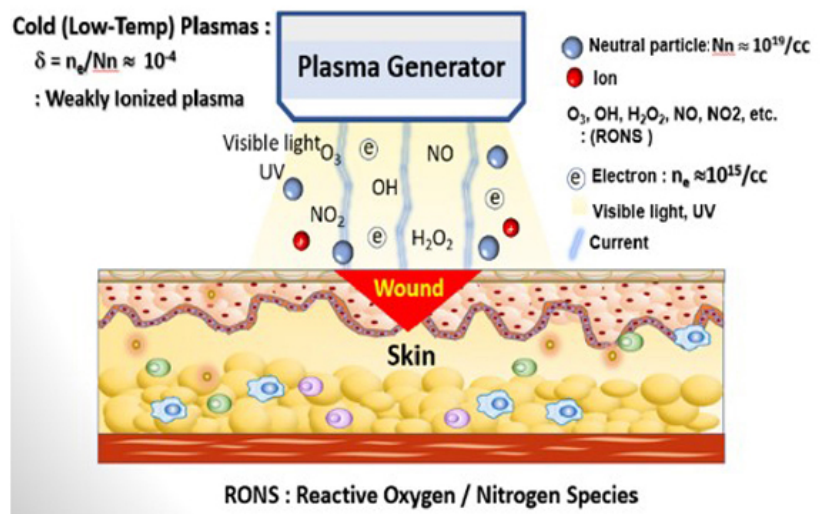

(b)

Figure 1. (a) Large area surface discharged NBP source for plasma biosciences and reactive oxygen and nitrogen species (RONS) in plasma bioscience. (b) Plasma RONS interactions with biological tissue. Reproduced with permission from [11] Copyright 2021, Springer Nature.
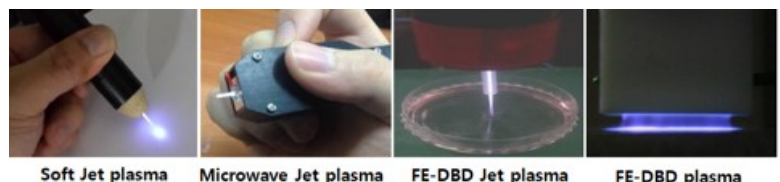

Soft Jet plasma

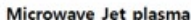

FE-DBD Jet plasma

FE-DBD plasma
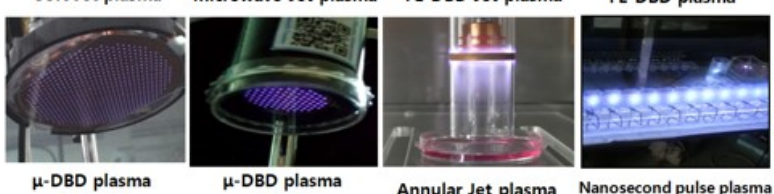

$(90 \mathrm{~mm})$

$(35 \mathrm{~mm})$

Annular Jet plasma

Nanosecond pulse plasma

Figure 2. Nonthermal atmospheric pressure biocompatible plasma (NBP) sources for plasma bioscience. Reproduced with permission from [4], Copyright 2015, Korean Vacuum Society.

and fisheries, beauty, especially coronavirus quarantine and treatment devices [3-5].

\subsection{Plasma jet}

Figure 3(a) displays the plasma jet system which is consisting mainly of a high voltage driving power supply, electrodes covered with dielectrics. A plasma jet device is assembled with a syringe and a glass or ceramic tube. This plasma device is an ac-driven jet with a few kilovolts of duty-cycled sinusoidal waves at a frequency of $60 \mathrm{~Hz}-$ $100 \mathrm{kHz}$ [81]. The clinical needle and syringe are used for guiding the gas flow. The inner electrode is made of stainless steel whose inner diameter is $1.2-2 \mathrm{~mm}$ and a thickness is $0.2-0.3 \mathrm{~mm}$, which is tightly 

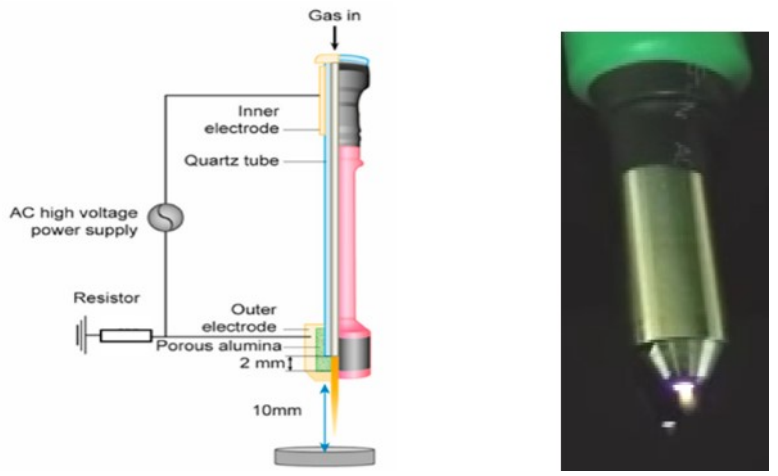

Figure 3. (a) A nonthermal soft plasma jet comprising mainly of a high-voltage power supply, dielectrics, and electrodes. Distance between the cell media or target and outer electrode was kept to 5-10 mm during exposure. Reproduced with permission from [81,82], Copyright 2020, MDPI. (b) Plume of soft plasma jet through exit nozzle. Reproduced with permission from [83], Copyright 2019, MDPI.

surrounded by a quartz tube whose outer diameter is $7-9 \mathrm{~mm}$. This serves also as a powered high voltage electrode in an alternative current. The glass or quartz tube covering the needle steers the flow of the plume. The glass tube also prevents electric shocks caused by high voltage naked needles during living tissue treatment. Here, the ground electrode is placed at the region of the glass tube end. The influence on the characteristics of the plasma plume by the ground electrode is important concerning the position and structure of the electrode. The primary voltage of the high voltage inverter is duty-cycled by a voltage timing controller. The outer electrode is fabricated from steel and it is perforated with $1 \mathrm{~mm}$ hole, via which the plasma jet is discharged into the neighboring ambient air. Porous alumina with a diameter of 10-12 $\mathrm{mm}$ and a length of $17-20 \mathrm{~mm}$ is machined to be in close contact with the outer electrodes and inner electrodes. The discharge gap distance is adjusted to $2-3 \mathrm{~mm}$ between the outer and inner electrodes. Gas is injected into the needle and then ejected via the 1-2 $\mathrm{mm}$ gap in the outer electrode via the porous alumina. The porous alumina utilized in this study is around $30 \mathrm{vol} \%$ porosity with an average pore diameter of $80-100 \mu \mathrm{m}$. Air or any other gases could be utilized as the feeding gas, the flow rate is controlled by a mass flow controller or an analog.

Once the gas is introduced via the inner electrode and ac power (high-voltage) from the inverter is applied, a plasma discharged into the porous alumina between the two electrodes, and a plasma jet achieving long lengths (up to several centimeters) is ejected into the open atmosphere, as demonstrated in Fig. 3(b) [83]. Cells or biological targets were treated with jet plasma for appropriate exposure times such as $30,60,120$, and $240 \mathrm{~s}$ under a discharge power of less than about 2 $\mathrm{W}$ (V peak: 1-2 kV and I 30sp : 1-2 mA) from a duty-cycle controlled inverter voltage. The driving frequency is $20-50 \mathrm{kHz}$ and $1-2 \mathrm{~L} / \mathrm{min}$ flow rate of air gas is maintained. The operating temperature of the plasma device is in the range of $25-36^{\circ} \mathrm{C}$ at the time of treatment.

\subsection{Surface type of dielectric barrier discharged non-} thermal atmospheric pressure plasma (surface DBD plasma)

Figure 4(a) shows a surface dielectric barrier discharged (DBD) nonthermal atmospheric pressure plasma for biosciences, medicines, and esthetics [84]. Biological skins have been treated by the surface DBD for $30 \mathrm{~s}$ to few minutes under electrical discharge power of about less than $3 \mathrm{~W}(1-2.2 \mathrm{kV}, 1-2 \mathrm{~mA}$, and phase angle 0.7 radians) with duty-cycle controlled high voltage ac inverter. The driving frequency of sinusoidal high-voltage could be controlled to be $60 \mathrm{~Hz}-100 \mathrm{kHz}$ as well as its duty cycles $1-50 \%$. The plasma device consisted of two parallel silver electrodes which are printed on the same insulating substrate. The coplanar two electrodes were separated by a $100-200 \mu \mathrm{m}$,

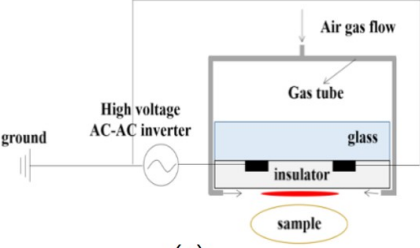

(a)

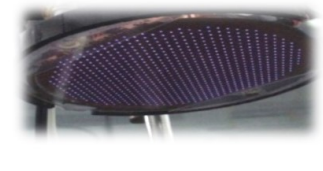

(b)
Figure 4. (a) Schematic of surface type of nonthermal atmospheric pressure dielectric barrier discharged (surface DBD) plasma system. Reproduced with permission from [84], Copyright 2020, MDPI. (b) Surface discharged image from DBD plasma. Reproduced with permission from [4], Copyright 2015, Korean Vacuum Society.

whose thickness is about 3-5 $\mu \mathrm{m}$, and tightly sealed with an insulating $\mathrm{SiO}_{2}$ paste. These electrodes are coated by Ag paste film by semiconductor screen printing or photolithography method with furnace treatment under $600{ }^{\circ} \mathrm{C}$ on the glass or ceramic substrate. The thickness of the substrate is about $1.8 \mathrm{~mm}$. The diameter of micro-DBD surface plasma is $30-100 \mathrm{~mm}$, which is designed for $20-100 \mathrm{~mm}$ Petridishes. The discharge power is about less than $3 \mathrm{~W}$ whose peak voltage is about $\mathrm{V}_{\mathrm{p}} \sim 1-2 \mathrm{kV}$ and currents are less than $5 \mathrm{~mA}$. The operating temperature of the plasma device is in the range of $24-32{ }^{\circ} \mathrm{C}$ at the time of treatment [85].

Once the gas is introduced through the peripheral guiding hole and high-voltage AC power from the inverter is applied, a discharge is fired onto the dielectric material as shown in Fig. 4(b), in which each spot represents respective discharged plasma whose thickness is limited to $\sim 200 \mu \mathrm{m}$ in plasma treatment $[4,84]$. Currently, surface DBD plasma devices with diameters of 35,90 , and $300 \mathrm{~mm}$ have been developed. It is applied to cancer cell death, skin care, wound and burn healing, agricultural and marine products storage technology, semiconductor surface treatment, and environmental improvement such as particulate mass dust removal.

Figure 5(a) shows the appearance of the counter-facing electrode's large-area surface discharged DBD plasma with a diameter bigger than $100 \mathrm{~mm}$, and Fig. 5(b) shows a schematic structure of counter facing electrode of this device [85]. These plasmas interact with ambient plasma gas, air or other buffer gases, and moisture in the atmosphere to generate various reactive oxygen species and nitrogen species. The low-temperature atmospheric pressure DBD plasma generated here has an electron temperature of $0.7-3 \mathrm{eV}$, and a plasma ion density of $(3-5) \times 10^{13-16} / \mathrm{cm}^{3}$ [85-88]. The density of various reactive species generated by the plasma of such an electron temperature has a value of approximately $(2-7) \times 10^{14-16} / \mathrm{cm}^{3}$ [85-88], and brings biological and medical effects. From a driving frequency of several $\mathrm{kHz}$ to several $\mathrm{MHz}$ and microwave of $2.45 \mathrm{GHz}$, it can generate plasma jets and large-area plasma in the form of the surface discharge as shown in Fig. 6(a) [47]. When such plasma is irradiated on the skin or lesion tissue, as shown in Fig. 6(b), simultaneous reactive species is generated in the cell tissue by plasma-initiated UV photolysis (red), in addition, the reactive gases generated at outside the cell tissue moves slowly (black) into the fine tissue due to the diffusion of transport $[89,90]$.

By the generation and interaction of such plasma reactive gases inside and outside the cell tissue, it is possible to heal cancer cells and various lesions on or in the skin tissue. At this time, a nonthermal large-area surface DBD plasma device can be used for large-area wound treatment and medical applications. In addition, standardization work on electrical safety, density, electron temperature, electrical energy, types and density of reactive oxygen species and nitrogen species, plasma treatment time of surface DBD-NBP device is also very important for their medical use. In particular, it is important to maintain the ozone content less than $0.05 \mathrm{ppm}[79,80]$, even though it is not international standards. In addition, $\mu$-DBD surface discharge plasma devices can be installed facing each other to produce a counter dis- 


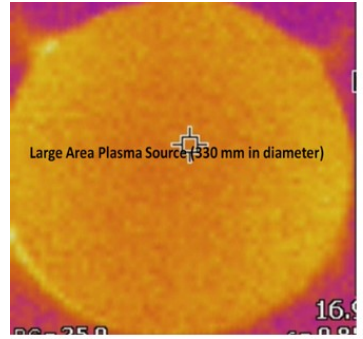

(a)

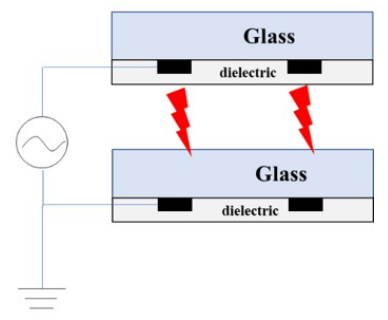

(b)
Figure 5. (a) Nonthermal facing large area DBD plasma source images with 330 $\mathrm{mm}$ in diameter. (b) The electrode structure of nonthermal facing large area DBD sources with $330 \mathrm{~mm}$ in diameter.

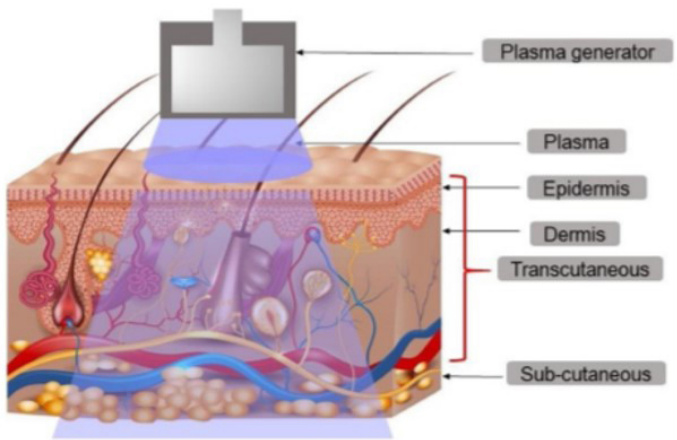

(a)

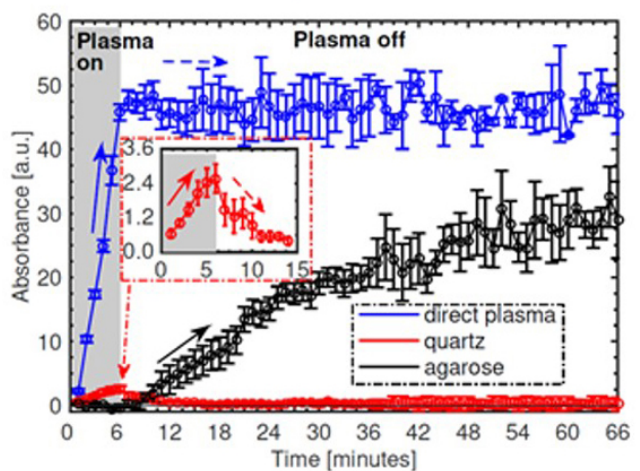

(b)

Figure 6. (a) Nonthermal atmospheric pressure plasma produces reactive oxygen and nitrogen species propagating the cutaneous materials through to subcutaneous layers underneath. Reproduced with permission from [47], Copyright 2019, Elsevier (b) Plasma induced UV photolysis produces simultaneous interior RONS generation in tissue and slow transport of exterior RONS into tissue. Reproduced with permission from [89], Copyright 2019, AIP Publishing.

charge electrode structure, and this device is being developed for use as a pathogen sterilizer for ambulances. It was proved plasma is very useful for sterilization and disinfection of ambulances using this when the corona crisis has recently expanded at Daegu, Korea in 2020, through an inactivation test of COVID19 [91].

\section{Diagnostics of nonthermal atmospheric pres- sure DBD plasma}

3.1. Electrical discharge voltage and current measurement

An oscilloscope could be used for the measurement of voltage and current during the discharge between the electrodes of nonthermal atmospheric pressure plasma. As shown in Fig. 7, the voltage could be

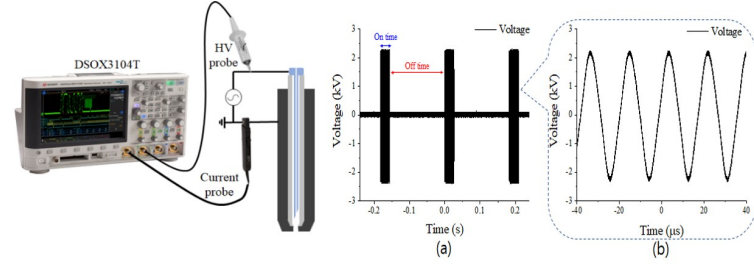

Figure 7. Electrical measurement of the plasma source. (a) An output voltage waveform of the DC-AC inverter with on-off time for soft plasma jet. (b) $2 \mathrm{kV}$ $50 \mathrm{kHz}$ sinusoidal wave. Reproduced with permission from [85], Copyright 2020 Springer.
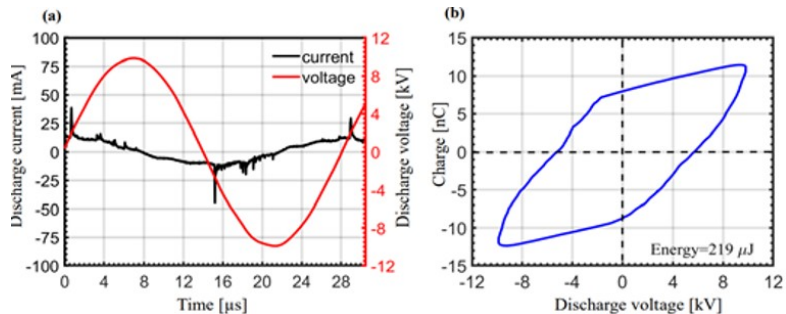

Figure 8. Electrical characteristics of discharge. (a) Discharge current (black) and voltage (red) waveforms versus time. (b) Plot of the corresponding charge versus voltage (Working gas: nitrogen; gas flow rate : 1,000 sccm). Reproduced with permission from [92], Copyright 2020, IOP Science.

measured between the HV (1000X) electrode and the grounded electrode, and the current could be monitored by a pick-up or Rogowski current probe. Figure 7(a) shows a driving voltage output (a) from the DC-AC inverter of a soft plasma jet, in which the duty-cycle ratio would be controlled for reduction of heat damage and ozone production during plasma discharge. The discharge of the soft plasma jet has been generated by about $\sim 2 \mathrm{kV}$ and $30-50 \mathrm{kHz}$ sinusoidal wave as in Fig. 7(b) under the nitrogen or any other gas flow rate of 1-2 $\mathrm{L} / \mathrm{min}[85]$.

The duration of on-time during discharge is adjusted by $15-25 \mathrm{~ms}$, and its duty ratio could be adjusted by $9-30 \%$ in the driving voltage by changing the off-time duration. The electrical energy per second could be obtained from the following equation with signal period $T$.

$$
\text { Electrical energy per second }=D * \frac{1}{T} \int_{0}^{T}(V(t) * I(t)) d t,
$$

where $D$ is the duty ratio defined by $\frac{T_{\text {on }}}{T_{\text {on }}+T_{\text {off }}}$, where $T_{\text {on }}$ and $T_{\text {off }}$ are on-time and off-time durations in driving voltage, respectively. The voltage (red) and current (black) waveforms used for the soft jet are shown in Fig. 8(a), respectively [92]. Positive discharge for soft jet occurs at the breakdown voltage of $2 \mathrm{kV}$ with a discharge peak current of $37 \mathrm{~mA}$, while a negative discharge occurs at the breakdown voltage of $-2 \mathrm{kV}$ with a discharge peak current of $-40 \mathrm{~mA}$, respectively, in which the voltage driving frequency has been measured to be $85 \mathrm{kHz}$. The electrical energy per unit cycle could also be obtained from the Lissajous figure, i.e., the charge versus applied voltage relationship, as shown in Fig. 8(b). The electrical energy per cycle is found to be 219 $\mu \mathrm{J}$ for a soft jet either from Eq. (1) or in Lissajous Fig. 8(b). The electrical discharge energy per second could be estimated to be $1.5 \mathrm{~J} / \mathrm{s}$ either from the Eq. (1) or from Lissajous figure under duty cycle $D=0.1$ and $f=85 \mathrm{kHz}$. This low power consumption is specific characteristics in nonthermal atmospheric pressure plasma for plasma biosciences and medicines.

\subsection{Optical emission spectroscopy measurement}

The optical emission spectroscopy could be performed to clarify reactive species by emission characteristics of the nonthermal atmospheric pressure plasma. For this kind of work, the spectrometer might 


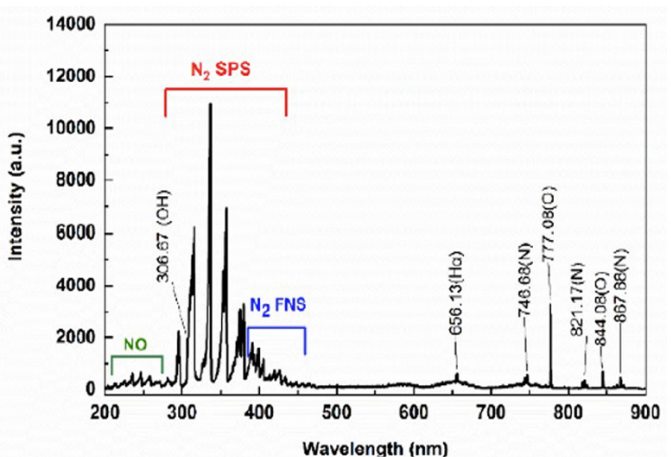

(a)

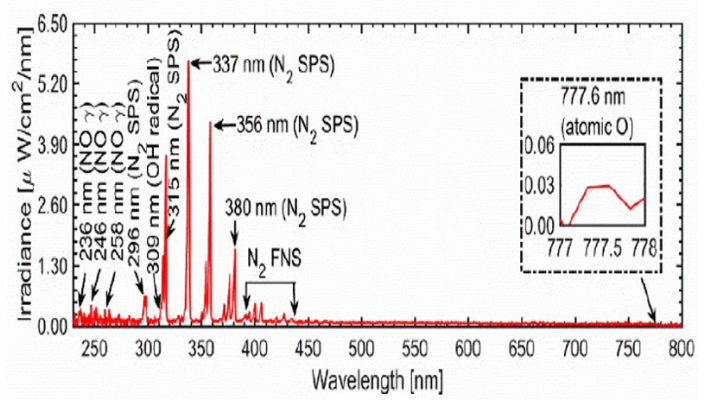

(b)

Figure 9. OES emission profiles from the soft plasma jet (a) and surface discharged $\mu$-DBD plasma (b), respectively. Reproduced with permission from [59], Copyright 2019, Springer Nature, [92] Copyright 2020, IOP Science, [93] Copyright 2020, Springer Nature.

be calibrated for wavelength measurements by using a Hg-Ar lamp. The opical emission spectra (OES) could be measured by using an optical fiber whose diameter is about $400 \mu \mathrm{m}$, placed in front of the nozzle of plasma plume, through which the emitted light is led to a slit for collecting the light into a grating of a spectrometer.

The OES of the soft plasma jet and surface discharged $\mu$-DBD have been represented in Figs. 9(a) and 9(b), respectively. There are discharge signals from the nitric oxide gamma band $(\mathrm{NO} \gamma)$ at 236, 246 and $258 \mathrm{~nm}$, etc [45] in both Figs. 9(a) and 9(b). These are caused by the collision of energetic electrons or metastable atoms with nitrogen molecules in the air. Also, the emission in the range of 306-309 nm could be observed, which are caused by hydroxyl radical $(\mathrm{OH})$ species by dissociation of water molecules being existing in the ambient environment [93]. The nitrogen second positive system $\left(\mathrm{N}_{2}\right.$ SPS) could be strongly observed at $296,315,337,356$, and $380 \mathrm{~nm}$, etc, moreover, the nitrogen first negative system $\left(\mathrm{N}_{2}\right.$ FNS) emissions are weakly observed at wavelengths ranged $390-440 \mathrm{~nm}[59,92,93]$. These emissions originated from the excited nitrogen species could be caused by nitrogen molecules both in the feeding gas and ambient environment. In addition to these, there are also emissions from the atomic oxygen (O) at 777 and $844 \mathrm{~nm}$, and emission at $656 \mathrm{~nm}$ from hydrogen atom $\left(\mathrm{H}_{\alpha}\right)$.

\subsection{Plasma electron temperature and electron den- sity measurement}

\section{Plasma electron temperature measurement}

In this section, we could study the plasma propagation speed, $u_{g}$, which may be described as plasma group velocity, by employing plasma fluid equation concerning ambipolar diffusion, which yields an equa- tion $u_{g} \approx c_{s}^{2} / u_{n}[60,61]$, where $c_{s}=\left(\frac{k T_{e}}{M_{i}}\right)^{1 / 2}$ is ion-acoustic wave velocity, $u_{n}=-D_{a} \nabla n / n$ is plasma ambipolar diffusion velocity, and $D_{a}=$ $c_{s}^{2} / v_{i}$ is the ambipolar diffusion coefficient. Herein $k, \nabla n, T_{e}, M_{i}, v_{i}$, and $n\left(\approx n_{e} \approx n_{i}\right.$ ) are Boltzmann constant, plasma density gradient, electron temperature, ion mass, ion-neutral collision frequency, and plasma density, respectively. Also, the $v_{i}$, in a weak and moderate electric field can be expressed in [62] by,

$$
v_{i}=\frac{e P \sqrt{\left(\alpha / a_{o}^{3}\right) A}}{2.7 M_{i} \sqrt{\left(1+\left(M / M_{i}\right)\right.}}
$$

where $P$ is gas pressure in Torr, $e$ is electron charge, $a_{0}$ is Bohr radius, $A$ is molecular weight of gas, $\alpha$ is polarizability of the molecules, and $M$ is mass of neutral gas. Since electrons and ions could not move independently due to relative high value of polarizing electric field, $E_{\text {polar }}$, they may tend to move mutually under velocity closely to ambipolar diffusion velocity, $u_{n}$. In this steady state condition moving at plasma bullet frame, the steady state continuity equation could be written by

$$
\frac{\partial^{2} n}{\partial x^{2}}+\frac{u_{g}}{D_{a}} \frac{\partial n}{\partial x}+\frac{v_{i z}}{D_{a}} n=0 .
$$

From this Eq. (2) the plasma ambipolar diffusion velocity $u_{n}$ could be solved from $u_{n}=-D_{a} \nabla n / n$ by after simple mathematical calculation [61],

$$
u_{n}=\frac{u_{g}}{2}-\frac{1}{2} \sqrt{u_{g}^{2}-\frac{4 v_{i z} k_{B} T_{e}}{M_{i} v_{i}}} .
$$

The electron temperature, $T_{e}$, can be determined by equating this formula Eq. (3) to

$u_{n} \approx c_{s}^{2} / u_{g}$ [24], by finding the following analytical equation.

$$
\frac{u_{g}}{2}-\frac{1}{2} \sqrt{u_{g}^{2}-\frac{4 v_{i z} k_{B} T_{e}}{M_{i} v_{i}}}=\frac{k_{B} T_{e}}{M_{i} u_{g}} .
$$

where the stepwise ionization frequency $v_{i z}$ could be written [Fridman] as

$$
v_{i z} \approx v_{i d}\left(I / T_{e}\right)^{7 / 2}
$$

Here $v_{i d}$ is direct ionization frequency given [62] by

$$
v_{i d} \approx N \sqrt{\frac{8 k_{B} T_{e}(\mathrm{~K})}{\pi m_{e}}} C_{i}\left(I+2 T_{e}(\mathrm{eV})\right) \exp \left(\frac{-I}{T_{e}(\mathrm{eV})}\right),
$$

where $N$ is neutral gas density $2.5 \times 10^{25} \mathrm{~m}^{-3}, m_{e}$ is electron mass, $C_{i}$ is gas constant, for instance $C_{i}$ of argon is $2 \times 10^{-17} \mathrm{~cm}^{2} / \mathrm{eV}$ and $C_{i}$ of neon is $1.6 \times 10^{-18} \mathrm{~cm}^{2} / \mathrm{eV}$, and $I$ is ionization potential.

The electron temperature inside the nonthermal atmospheric pressure plasma can be achieved by plotting right and left-hand side terms of Eq. (4) in terms of electron temperature, as shown in Fig. 10, by obtaining a junction point where the right and left-hand side terms are crossed at a certain temperature. It can be observed in Fig. 2 that electron temperature of atmospheric pressure nonthermal plasma is detected to be about $1.18 \mathrm{eV}$ under the experimentally measured group velocity, $u_{g} \approx 4 \times 10^{4} \mathrm{~m} / \mathrm{s}$ as of many experiments around the world wide laboratories [25-39].

In this context, we assumed a hypothesis that nature will conserve the fluxes of electron and ion to be almost the same due to the density of ion and electron within the plasma are relatively elevated values and additionally this fact will give rise to the quasineutrality, $n_{i}$ $\approx n_{e} \approx n$. Therefore, the polarization electric field, $E_{\text {polar }}$, caused by the space charge inside nonthermal dielectric barrier discharged plasma would be ambipolar electric field and has a major impact to control and modify the resultant fluxes of ions and electrons to be approximately similar to each other [61]. Here we also note that this 


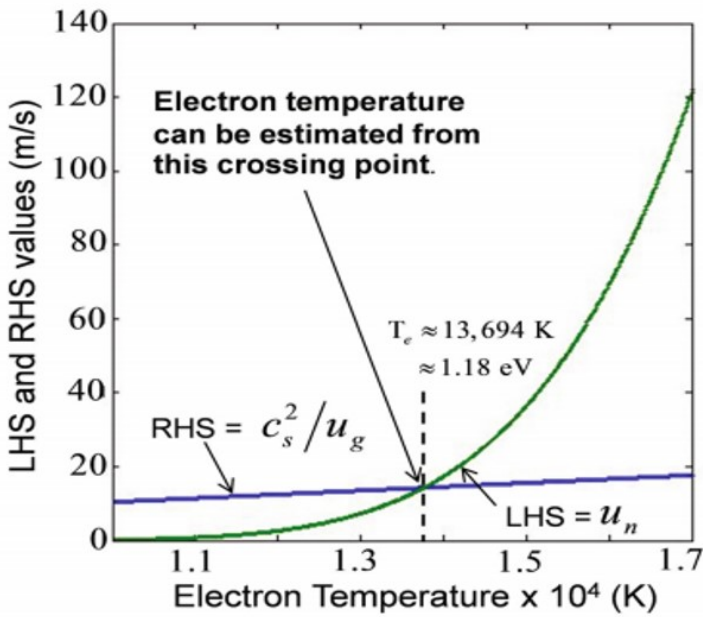

Figure 10. Determination of electron temperature by finding an intersection point of the left and right-hand side of Eq. (4) under measured plasma group velocity, $u_{g} \approx 4 \times 10^{4} \mathrm{~m} / \mathrm{s}$ from experiment by scanning the electron temperature from 0 to $1.7 \times 10^{4} \mathrm{~K}$. Reproduced with permission from [61], Copyright 2017, Springer Nature.

method for determination of electron temperature with stepwise ionization and convective wavepacket plasma model has many advantages in real applications and could be very widely and easily applied to either plasma jet and a large area of dielectric barrier surface discharged plasma without any loss of generality under various kinds of pure gases or their mixtures. However, the other methods such as the collisional radiative model have many limitations and are complicated to real situations in their measurement since there is no confirmative assumption of plasma equilibrium or local thermodynamic equilibrium state in nonthermal atmospheric pressure DBD plasma. The electron temperature for the soft jet and the surface $\mu$-DBD plasma has been measured to be 1.76 and $0.6 \mathrm{eV}$ based on the collisional radiative model $[11,47,63,85-88]$.

\section{Plasma electron density measurement}

For estimation of the electron density inside the nonthermal DBD plasma, we assume that both electron and ion will move together by ambipolar diffusion velocity. However, in actual conditions, it is very difficult for electron flux to be precisely the same as that of ion flux. Also, electron flux may be slightly greater than ion flux due to electron has higher diffusion coefficient, $D_{e}$ than that of ion diffusion coefficient, $D_{i}$ as well as $\mu_{e}>>\mu_{i}$, where $\mu_{e}$ is electron mobility and $\mu_{i}$, is ion mobility. Therefore, the current density might be expressed as $J \approx$ $e n u_{e}$, where $u_{e}$ is downstream electron velocity, with no violation of quasineutrality as $n \approx n_{e} \approx n_{i}$. From this justification, the downstream current density is not zero, i.e., $J \neq 0$, whenever we detected it in the experiment. The ambipolar electric field, $E_{\text {polar }}$, causes ambipolar diffusion for $\Gamma_{e} \approx \Gamma_{i}$, the downstream electron speed $u_{e}$ should be close to ambipolar diffusion velocity, $u_{n}$. Therefore, the current density in the downstream region could be expressed by

$$
J \approx e n u_{e} \approx e n u_{n},
$$

which is dominant electron flux under conditions of $\Gamma_{e} \approx \Gamma_{i}$. Therefore, the electron density could be saved and estimated by using Eq. (5) $[11,61]$. The peak value of downstream electron current has been measured to be about $I_{\text {peak }} \approx 12 \mathrm{~mA}$ with a plasma radius of $r_{b}=2.67$ $\times 10^{-4} \mathrm{~m}$ in the experiment [61]. Here the averaged current density $J$ can be written by $J=I_{\text {peak }} / 2 A$, where $A=\pi r_{b}{ }^{2}$ is the cross-sectional area through which the plasma has been passed. The electron density has then been estimated to be $3 \times 10^{15} \mathrm{~cm}^{-3}$ from Eq. (5) based on the ambipolar diffusion model [61]. The electron density from $\mu$ DBD jet and $\mu$-DBD surface plasma has been estimated to be $3 \times 10^{15}$ and $5 \times 10^{14} \mathrm{~cm}^{-3}$ from the ambipolar diffusion model, respectively. The electric field corresponding to this electron density is estimated by $E \sim \frac{n_{e} e \lambda_{D}}{\varepsilon_{0}}$, where $\lambda_{D}=740 \sqrt{\frac{k T_{e}[e V]}{n_{e}\left[\mathrm{~cm}^{-3}\right]}}(\mathrm{cm})$ is electron Debye length. This electric field has been approximately to be $12-70 \mathrm{kV} / \mathrm{cm}$ for $\mu$-DBD jet and $\mu$-DBD surface plasma, which is reported from many laboratories for $\mu$-DBD plasma [64]. This could play a very crucial role in biological interactions, especially in cell membrane interfacial region with water resulting in depolarization of membrane structure to induce apoptosis.

The other methods for the determination of electron density for nonthermal atmospheric pressure DBD plasma are Stark broadening [65] and laser interferometric spectroscopic diagnostics [66], in which their plasma densities are somewhat similar to that obtained from the convective wavepacket method introduced here. Hence we would like to suggest this kind of measurement of electron density in nonthermal atmospheric pressure DBD plasma since it is a relatively easy and economic facility in most laboratories.

\subsection{Rotational and vibrational plasma gas tempera- ture measurement}

Measurement of molecular gas temperature is fundamentally important for understanding the characteristics of the nonthermal atmospheric pressure DBD plasma. In nitrogen discharged plasmas, the rotational gas temperatures of nitrogen quickly achieve their thermodynamic equilibrium within themselves, due to the faster rotational energy exchanges with surrounding ambient heavy particles rather than with electrons. The Boltzmann distribution of rotational levels could be used to measure the rotational temperature in $\mu$-DBD plasmas [67, $68,87]$. Optical emission spectroscopy has been extensively used to estimate the rotational temperature in $\mu$-DBD plasmas $[67,68,87]$. Many people showed that the most accurate value of gas temperature could be obtained using the $\mathrm{N}_{2} \mathrm{C}^{3} I I_{u} \rightarrow \mathrm{N}_{2} B^{3} I I g$, so-called the second positive system (SPS) emission band $[67,68,87]$ rather than the first positive system (FPS) of $N_{2}+B^{2} \Sigma_{u}^{+} \rightarrow N_{2}+X^{2} \Sigma_{g}^{+}$, where their transition energy diagram and real emission spectra are shown in Fig. 11. Figure 11 shows the OES of the nonthermal DBD plasma jet, where $\mathrm{N}_{2}$ SPS (297-400 nm) and $\mathrm{N}_{2}$ FPS (550-800 nm) are observed, where NO- $\gamma$ band (213-258 nm) could also be observed [85]. The OES from the nonthermal $\mu$-DBD surface plasma is similar to that of the plasma jet. Vibrational spectra of the $\mathrm{N}_{2}$ SPS and their magnified rotational spectrum belonging to the $0-0$ vibrational band around $337 \mathrm{~nm}$ of $\mathrm{N}_{2}$ $C^{3} I I_{u}(v=0) \rightarrow N_{2} B^{3} I I_{g}(v=0)$, as indicated by dotted box in (b).

In this work, we have selected rotational levels of the SPS belonging to the $0-0$ vibrational band of $N_{2}\left(C^{3} \Pi_{u}\right)(v=0) \rightarrow B^{3} \Pi_{g}(v=0)$, as indicated by magnified spectrum around $337 \mathrm{~nm}$, in Fig. 11(b). The intensity $I_{J^{\prime} J^{\prime \prime}}$ of a particular rotational transition $J^{\prime} \rightarrow J^{\prime \prime}$ in this SPS is given by $[53,54]$

$$
I_{J^{\prime} J^{\prime \prime}} \approx A_{v^{\prime} v^{\prime \prime}} S_{p}\left(J^{\prime \prime}\right) n_{c} \exp \left(-\frac{E_{r}\left(v^{\prime}, J^{\prime}\right)}{k T_{\text {rot }}}\right)
$$

where $A_{v^{\prime} v^{\prime \prime}}$ is transition amplitude, $S_{p}\left(J^{\prime \prime}\right)=6 J^{\prime \prime}-10 / J^{\prime \prime}$ is HoenlLondon factor corresponding to the rotational angular momentum quantum number $J^{\prime \prime}$, and $E_{r}\left(v^{\prime}, J^{\prime}\right)$ is the rotational energy for vibrational number $v^{\prime}$ and rotational number $J^{\prime}$ given by

$$
E_{r}\left(v^{\prime}, J^{\prime}\right)=B_{v} J^{\prime}\left(J^{\prime}+1\right)-D_{v} J^{\prime}\left(J^{\prime}+1\right)^{2}
$$

where $B_{v}$ and $D_{v}$ are rotational term for vibrational number $v, J^{\prime}$ and $J^{\prime \prime}$ are the rotational quantum numbers for the transitions ( $v=0$, $\left.J^{\prime}\right) \rightarrow\left(v=0, J^{\prime \prime}\right), k$ is the Boltzmann constant, and $T_{r o t}$ is the rotational temperature. For the estimation of rotational temperature, Eq. (6) 


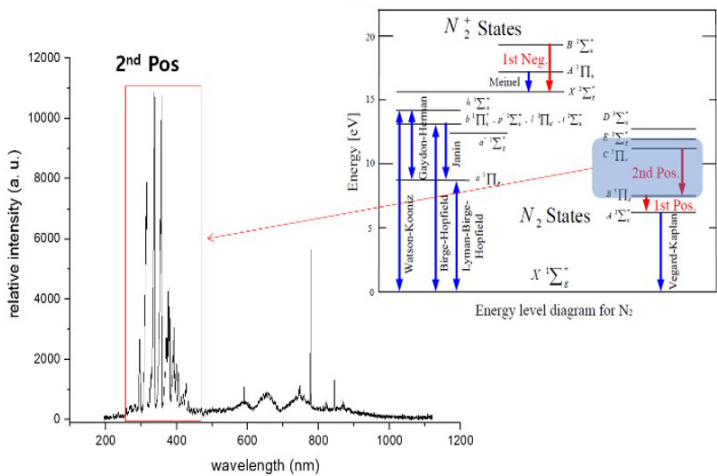

(a)

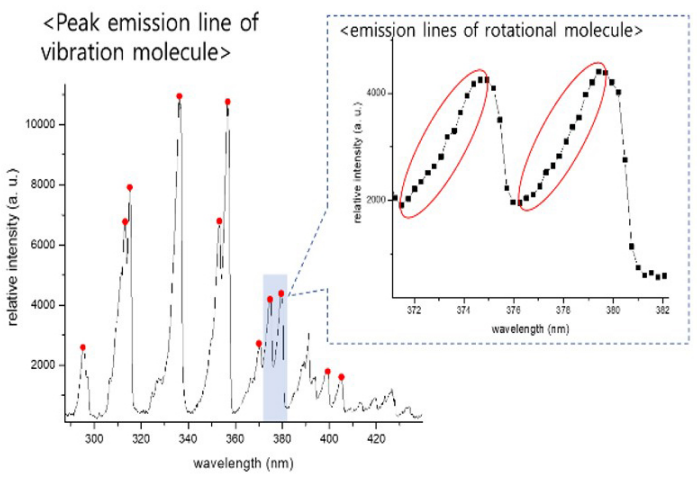

(b)

Figure 11. (a) Optical emission spectra and their transitional energy diagram $\mathrm{N}_{2}$ $C^{3}\left\|I_{u} \rightarrow B^{3}\right\| I_{g}$, the second positive system (SPS). (b) Vibrational spectra of the $\mathrm{N}_{2}$ SPS and magnified rotational spectrum belonging to the $0-0$ vibrational band around $337 \mathrm{~nm}$ of $\mathrm{N}_{2} C^{3}\left\|_{u}(v=0) \rightarrow B^{3}\right\|_{g}(v=0)$, as in dotted box in (b). Reproduced with permission from [85], Copyright 2020, Springer Nature.

could be rearranged as

$$
\ln \left(-\frac{I_{J^{\prime} J^{\prime \prime}}}{S_{p}\left(J^{\prime \prime}\right)}\right)=-\frac{B_{v}^{\prime} h c}{k T_{\text {rot }}} J^{\prime}\left(J^{\prime}+1\right)+C
$$

where $h$ is Plank constant, $c$ is the speed of light, and $C$ is constant. Figure 12 shows the plot of Eq. (8) versus rotational energy given by Eq. (7) with rotational quantum number $J^{\prime}$. The rotational temperature $T_{\text {rot }}$ of the plasma gas molecules could be determined from the reciprocal value of the slope of the resulting linear plotting of the lefthand side of Eq. (8) versus the energy difference between rotational energy and $E_{r}$. The slope of the resulting linear distribution will be equal to $B^{\prime}{ }_{v} h c /\left(k T_{r o t}\right)$. From the reciprocal value of this slope, the rotational temperature $T_{\text {rot }}$ of the plasma gas has been measured to be $807 \mathrm{~K}$ for nonthermal air plasma plume above $1 \mathrm{~mm}$ water surface throughout repeated experiments.

In this work, the vibrational gas temperature could also be estimated by selecting quantum levels of the SPS corresponding to the $v$ $v^{\prime}$ vibrational band of $N_{2}\left(C^{3} \Pi_{u}\right)(v) \rightarrow B^{3} \Pi_{g}\left(v^{\prime}\right)$. The intensity of a particular vibrational band in this SPS is given by [69],

$$
\ln \left(\frac{I_{v v^{\prime}}}{v_{v v^{\prime}} A_{v v^{\prime}}}\right)=C-\frac{E_{v}-E_{0}}{k T_{v i b}}
$$

where $I_{v v^{\prime}}$ is emission intensity transition $v \rightarrow v^{\prime}$, measured from experiment, $v_{v v^{\prime}}$ is transitional frequency, $A_{v v^{\prime}}$ is transitional amplitude, and $E_{v}$ is the vibrational energy for quantim number $v$ given by

$$
E_{v}(\mathrm{eV})=1.24 \times 10^{-4}\left(\frac{1}{2}+v\right)\left[\omega_{e}(\mathrm{~cm})^{-1}\right]
$$

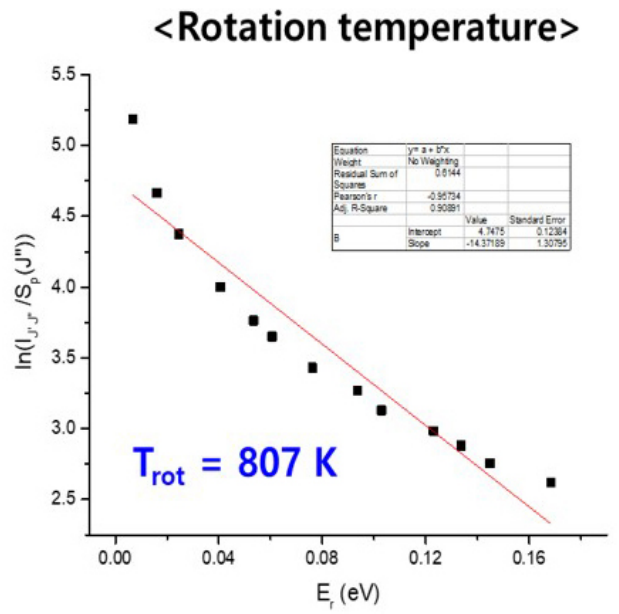

(a)

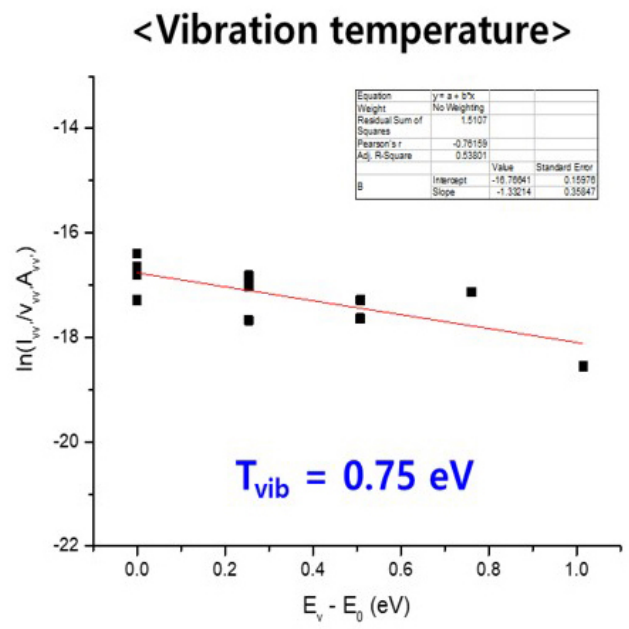

(b)

Figure 12. Rotational and vibrational gas temperatures of nonthermal air DBD plasma plume could be determined from the inverse slope of the above figure (a) and (b), respectively, which yield $807 \mathrm{~K}$ and $0.75 \mathrm{eV}$, at $1 \mathrm{~mm}$ above the position of water surface. Reproduced with permission from [85], Copyright 2020, Springer Nature.

The vibrational temperature of $T_{v i b}$ of the plasma molecules could be determined from the reciprocal value of the slope of the resulting linear plotting of the left-hand side of Eq. (9) versus energy difference $E_{r}-E_{o}$. The slope of the resulting linear distribution will be equal to $1 /\left(k T_{v i b}\right)$. From the reciprocal value of this slope, the vibrational temperature $k T_{v i b}$ of the plasma gas has been measured to be $0.75 \mathrm{eV}$ for nonthermal air plasma plume above $1 \mathrm{~mm}$ water surface, as indicated by Fig. 12(b) [87]. Since the maximum exciting nitrogen number density among the excited molecular energy band $C, B$, and $A$ are up to $\sim 10^{17} / \mathrm{cm}^{-3}$ in this experiment, the ratio of excited molecules of rotational and vibrational temperature $807 \mathrm{~K}$ and $0.75 \mathrm{eV}$, respectively, to those of cooled ambient molecules is estimated to be about $1 \%$, in which remaining $99 \%$ of most ambient molecules with room temperature are quickly absorbing rotational and vibrational temperatures of the excited $1 \%$ those molecules, resulting in cool temperature if your hand is touching to this DBD plasma. Hence we call this kind of plasma nonthermal, cold, or cool plasma even though the rotational temperature is somewhat high to be $806 \mathrm{~K}$. 


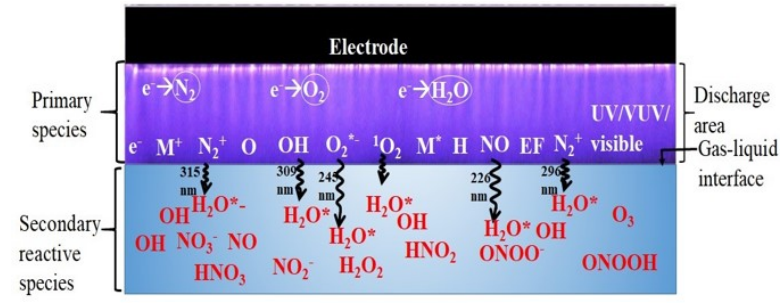

Figure 13. Schematics for electrons, ions, and neutral particles along with VUV and UV radiation contribute to the production of the reactive oxygen and nitrogen (RONS) species in discharge areas in gas and water interior region during the plasma discharge. Reproduced with permission from [47], Copyright 2019, Elsevier.

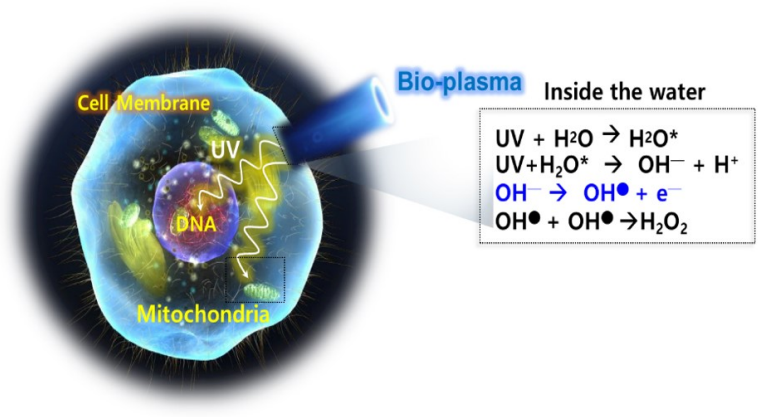

Figure 14. Plasma-based UV photolysis: Generation mechanism of ROS inside the water and biological cell or tissue. Reproduced with permission from [4], Copyright 2015, Korean Vacuum Society, [12] Copyright 2015, Springer Nature.

3.5. Plasms initiated ultraviolet photolysis for reactive species near to water surface and interior region of the water

Water is a good model for the understanding of the physics and chemistry that occurred in the plasma-induced reactive species near to water surface and propagation of them into water. If nonthermal plasma has interacted with the water, there are many physics and chemistry issues $[70,88]$ are involved during their interactions. Water is the simplest environment for investigating plasma-liquid interactions even in the living tissue. Plasma interfacial interactions with water generate highly reactive molecules such as the $\mathrm{O}_{2}{ }^{*-}, \mathrm{OH}$, $\mathrm{NO}$ and $\mathrm{ONOO}^{-}$and others.

Figure 13 shows how electrons, ions, and neutral particles along with VUV and UV radiation contribute to the production of the RONS species in discharge gas and water interior region during the plasma bombardment on the water [47]. The RONS production mechanism of plasma initiated ultraviolet photolysis interior region of water and diffusion process into the water during the plasma discharge on the water. The electrons in discharged gas or interfacial region near to water surface could collide with neutral molecular gases of nitrogen, oxygen, water vapor, etc and dissociate them resulting in primary RONS species such as $\mathrm{OH} \cdot \mathrm{H}_{2} \mathrm{O}_{2}, \mathrm{O}, \mathrm{O}_{2}{ }^{*-},{ }^{1} \mathrm{O}_{2}, \mathrm{O}_{3}, \mathrm{~N}_{2}{ }^{+}, \mathrm{NO}$, and $\mathrm{NO}_{2}$ in the discharge gas region. These primary reactive species initiate UV light ranged from 190 to $\sim 400 \mathrm{~nm}$, caused by electron impacts to nitrogen, oxygen, and water molecules, which propagates into the water to excite water molecules for simultaneous production of $\mathrm{OH} \cdot$ and $\mathrm{NO}$ at the interior region of water.

Figure 14 shows plasma initiated ultraviolet photolysis, which is a generation mechanism of RONS species inside the water and biological cell or tissue [12]. Their main reactions for RONS production inside the water during the plasma discharges are done on the water surface [12],

$$
\begin{aligned}
& \mathrm{UV}+\mathrm{H}_{2} \mathrm{O} \rightarrow \mathrm{H}_{2} \mathrm{O}^{*} \\
& \mathrm{UV}+\mathrm{H}_{2} \mathrm{O}^{*} \rightarrow \mathrm{OH} \cdot+\mathrm{H}^{*}, \text { or } \mathrm{O}+2 \mathrm{H}
\end{aligned}
$$

$$
\begin{aligned}
& \mathrm{OH} \cdot+\mathrm{OH} \cdot \rightarrow \mathrm{H}_{2} \mathrm{O}_{2} \text {, or } \mathrm{H}_{2} \mathrm{O}+\mathrm{O} \\
& \mathrm{H}_{2} \mathrm{O}^{*} \rightarrow \mathrm{OH}^{-}+\mathrm{H}^{+} \\
& \mathrm{OH}^{-}+\mathrm{OH}^{-} \rightarrow \mathrm{H}_{2} \mathrm{O}_{2}+2 \mathrm{e}^{-} \\
& \mathrm{OH}^{-} \rightarrow \mathrm{OH} \cdot+\mathrm{e}^{-} \\
& \mathrm{O}_{3}+\mathrm{OH}^{-}+\mathrm{H}^{+} \rightarrow 2 \mathrm{OH} \cdot+\mathrm{O}_{2}
\end{aligned}
$$

in which the reaction of Eq. (16), $\mathrm{OH}^{-} \rightarrow \mathrm{OH} \cdot+\mathrm{e}^{-}$would also play the crucial role in the generation of $\mathrm{OH}$ radicals since it consumes very little energy $\approx 2.4 \mathrm{eV}$, which could be explained for $\mathrm{OH}$ generation in the deeper water or biological tissue. Consequently these leads to $\mathrm{H}_{2} \mathrm{O}_{2}$ and $\mathrm{NO}_{2}$ production in the water $[12,47,89,90,92,94]$. The primary RONS generated by electron impact in discharge gas or interfacial region along with the secondary RONS by plasma-initiated UV photolysis at interfacial or water interior region is both starting to be diffused into the water. Additional $\mathrm{HO}_{2}$ and $\mathrm{NO}_{2}$ radicals are produced through reactions of $\mathrm{OH}$. and $\mathrm{NO}$ with secondary RONS. It is very difficult to analyze changes in liquid chemistry at different depths by sufficient spatiotemporal resolution and accuracy. In most cases, it is very difficult to investigate a full picture of plasma physics and chemistry of water, especially plasma RONS production in water are remaining very intensive research activities. Nevertheless, significant advances in the understanding of some mechanisms have been achieved and summarized [94,95]. Plasma diagnostics and analytical chemistry for plasma-liquid interactions have been studied both experimentally and in mathematical models.

Additionally, RONS generation also has been influenced by plasma electric fields, environmental humidity, and gas composition. The RONS concentrations would be adjusted by discharge voltages, gas species, and its flow rate as well as plasma operating times [96-98]. Also, the OES intensity emitted from $\mathrm{Ar}-\mathrm{O}_{2}$ plasma could be used for achieving information of all RONS species in their gas environments. Their intensities are decreased as compared with pure Ar plasma since the addition of $\mathrm{O}_{2}$ decreases the number of discharging electrons due to its electronegative characteristics. In the water, the concentrations of $\mathrm{H}_{2} \mathrm{O}_{2}$ and $\mathrm{OH}$ would be decreased for $\mathrm{Ar}-\mathrm{O}_{2}$ and $\mathrm{Ar}-\mathrm{N}_{2}$ plasma in comparison with those of pure Ar plasma. While the concentration of $\mathrm{NO}_{2}{ }^{-}$and $\mathrm{NO}_{3}{ }^{-}$is measured to be high for the Ar- $\mathrm{N}_{2}$ plasma compared with the other plasmas [98]. The admixtures plasma with $\mathrm{He}+\mathrm{O}_{2}+\mathrm{H}_{2} \mathrm{O}$ is enabled to generate various ROS species such as $\mathrm{O}$, $\mathrm{O}_{2}{ }^{*}, \mathrm{O}_{3}, \mathrm{OH}$, and $\mathrm{H}_{2} \mathrm{O}_{2}$ [99] under the conclusion that ROS for this plasma is unaffected by the ratios of $\mathrm{H}_{2} \mathrm{O} / \mathrm{O}_{2}$ in the feeding gas. Also for maximum production of $\mathrm{OH}$ and $\mathrm{O}$ simultaneously, the ratios of $\mathrm{H}_{2} \mathrm{O} / \mathrm{O}_{2}$ in the feeding gas is found to be $\approx 1$. Although it is also found that a higher concentration of $\mathrm{H}_{2} \mathrm{O}_{2}$ could be achieved than the $\mathrm{NO}_{2}{ }^{-}$when the plasma plume is reached to the water surface. However, if this is the case when the plasma plume is not in contact with liquid, then the higher concentration of $\mathrm{NO}_{2}{ }^{-}$is achieved [100]. Further, many studies show that the $\mathrm{H}_{2} \mathrm{O}_{2}$ concentration depends strongly upon the humidity in feeding gas, rather than upon the distance of the plasma jet from the liquid surface [101].

This review shows various aspects of the reactive species during plasma exposures on water surface. During the discharge, the UV plays an important role to generate the reactive species inside the biological cell or tissues. The concentration of reactive species in the discharge gas phase, gas-liquid interface, and water interior during plasma exposures depends upon the distance between the plasma plume and liquid, plasma electric fields, humidity, gas mixtures, VUV, UV, gas flow rate, and duty cycle of operating voltage. Plasma-initiated UV photolysis $[94,102,103]$ also has a significant impact on the generation of $\mathrm{OH}$ - radicals and other RONS below the plasma-water interface, intercellular regions, or inside the tissue. The combined effects of plasma-initiated UV photolysis and transport of RONS into the cell or tissue would be responsible for the RONS production mechanism inside the water or biological cell and tissue [94, 102, 103]. The primary RONS generated by electron impact during discharge and the 
secondary RONS produced by plasma-initiated UV photolysis is both diffused into the water from their initial depths. These RONS are accumulated in water and surrounding cells, then consequently interacts with the cellular membrane that protects the intracellular environment.

\section{Diagnostics of hydroxyl radical $\mathrm{OH}$ species}

The spatial density distribution of RONS, especially for the hydroxyl radicals, i.e., $\mathrm{OH}$, could be diagnosed by the UV absorption spectroscopy from the nonthermal atmospheric pressure DBD plasma. The schematic experimental setup for the UV absorption spectroscopy could be found in many previous publications $[104,105]$. This system consists of UV lamp, which is a Hg lamp whose power is less than 1 $\mathrm{W}$ centered at a wavelength of $306 \mathrm{~nm}$, a plano-convex lens, and CCD spectrometer with optical fiber. The UV light has been passed and focused by $200 \mu \mathrm{m}$ on the plasma jet by the plano-convex lens for measurement of absorption occurred at $309.2 \mathrm{~nm}$, for the $\mathrm{OH}$ species. The hydroxyl $\mathrm{OH}$ radical density produced from air, argon, nitrogen or any kind of gas plasma could be investigated by using Lambert-Beer's law. The intensity for the incident and transmitted light passing through the plasma space whose thickness is $x$, are denoted to be $I_{o}$ and $I_{v}$, respectively. The density of hydroxyl radical $\mathrm{OH}$ species produced by the plasma jet is given by [104-106]

$$
N=-\frac{1}{\sigma \cdot x} \ln \left(\frac{I_{v}}{I_{0}}\right)
$$

where $N$ is the density of hydroxyl $\mathrm{OH}$ radicals, $\sigma$ is the molecular cross-sectional area about $6 \times 10^{-7} \mathrm{~cm}^{2}$ for $\mathrm{OH}$ species [106], and $x$ is $0.3 \mathrm{~cm}$. The hydroxyl radical $\mathrm{OH}$ density could be obtained by Eq. (18) from the experimental measurement of $I_{v} / I_{o}$, i.e., ratio of the transmitted intensity to incident one.

Figure 15(a) shows the UV absorption profile, represented by the black line, caused by the $\mathrm{OH}$ radical species for nonthermal Ar plasma jet whose gas flow rates are ranged from 80 to $300 \mathrm{sccm}$ [104], versus the wavelengths. Also, the reference UV lamp $\left(I_{o}\right)$ and emission profiles from $\mathrm{OH}$ radical species in the nonthermal plasma without UV incidence have been shown by red and blue lines, respectively, in this figure. The strong $\mathrm{OH}$ absorption profiles are appeared at the $309.2 \mathrm{~nm}$, as shown in the dotted box, while there is no absorption at $\sim 307 \mathrm{~nm}$. In this experiment, the absorbed signals that appeared at $\sim 317 \mathrm{~nm}$ is looking like to be nothing to do with $\mathrm{OH}$ radical species. The absorbed UV profiles (black line) in Fig. 15 could be obtained by subtracting the emission profiles of hydroxyl $\mathrm{OH}$ radical species (blue line) in plasma from the transmitted UV intensity $\left(I_{v}\right)$ passing through the atmospheric pressure plasm jet $[104,105]$. The UV emission and absorption profiles at $309 \mathrm{~nm}$ are denoted by the circles in the blue line and the dotted box in the black one, respectively, as shown in Fig. 15(a). The transmission ratio $\left(I_{v} / I_{o}\right)$ of UV lamp caused by hydroxyl $\mathrm{OH}$ radical species, from which the $\mathrm{OH}$ density or concentration could be estimated at an absorbed wavelength of $309 \mathrm{~nm}$. Figure 15(b) shows the $\mathrm{OH}$ radical density at $2 \mathrm{~mm}$ above the interfacial surface versus the Ar gas flow rate from 80 to $240 \mathrm{sccm}$ under the low electrical power of $15 \mathrm{~W}$ with the driving frequency of $22 \mathrm{kHz}$. The $\mathrm{OH}$ density reaches the maximum value of $2.6 \times 10^{15} \mathrm{~cm}^{-3}$ for the gas flow rate of $\sim 150$ sccm and it is rapidly decreased to $6.0 \times 10^{14} \mathrm{~cm}^{-3}$ for the flow rate of $\sim$ $250 \mathrm{sccm}$ in this experiment. Other groups also reported $\mathrm{OH}$ densities of $(0.3-7.5) \times 10^{15} \mathrm{~cm}^{-3}$ for low contents of water molecules less than $3 \%$ under operating gases of $\mathrm{He}, \mathrm{N}_{2}$, and $\mathrm{N}_{2} / \mathrm{O}_{2}$ mixtures [106-108] with microwave frequency $2.45 \mathrm{GHz}$ [107-109] and RF frequency of $13.56 \mathrm{MHz}$ [110] by electrical power bigger than $100 \mathrm{~W}$.

When the nonthermal atmospheric pressure DBD plasma touches the water surface, the $\mathrm{OH}$ density obtained increases drastically to 6.55 $\times 10^{16} \mathrm{~cm}^{-3}$ at an appropriate applied voltage [66]. This drastic increase in $\mathrm{OH}$ radicals could be explained by the dissociation of water

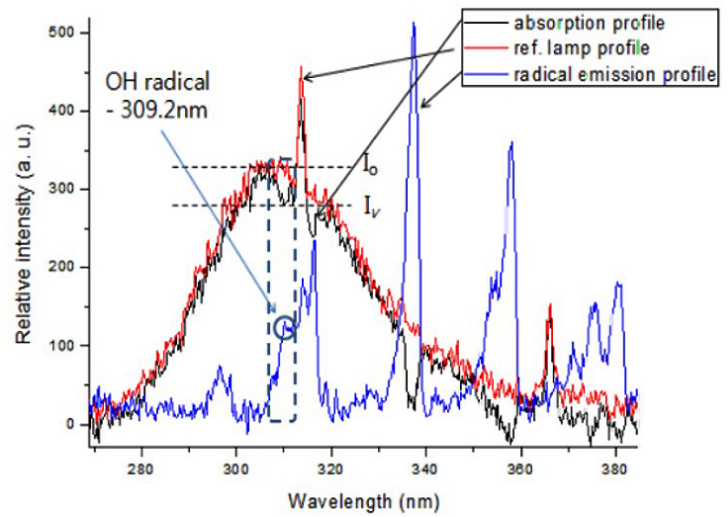

(a)

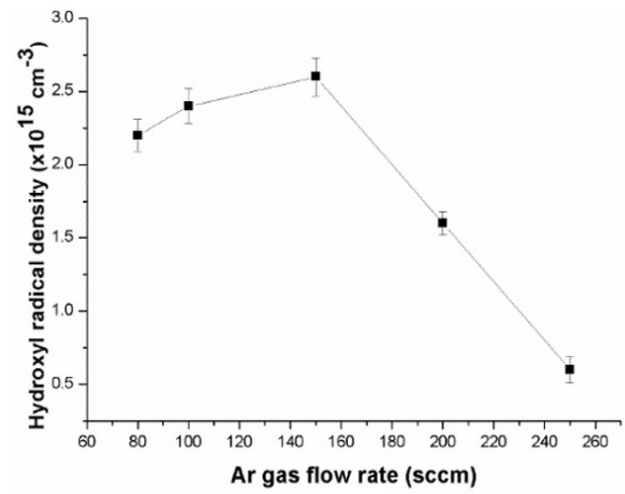

(b)

Figure 15. (a) UV absorption profile caused by the $\mathrm{OH}$ radical species (black) versus the wavelengths. Reference UV lamp profile $\left(I_{o}\right)$ versus the wavelength without nonthermal atmospheric pressure plasma jet (red). Emission profiles from the plasma versus the wavelength from hydroxyl $\mathrm{OH}$ radical species (blue) without UV incidence. (b) Hydroxyl OH radical density at the region of $2 \mathrm{~mm}$ above the interfacial surface, versus the Ar gas flow rate ranged from 80 to $240 \mathrm{sccm}$. Reproduced with permission from [104], Copyright 2012, IOP Science.

molecules by $\mathrm{Ar}$ atoms, $\mathrm{O}$ atoms, and electrons [111-114], in case $\mathrm{Ar}$ has been used for plasma generation, as follows:

$$
\begin{aligned}
& \mathrm{Ar}^{*}+\mathrm{H}_{2} \mathrm{O} \rightarrow \mathrm{OH}+\mathrm{H}+\mathrm{Ar} \\
& \mathrm{O}(1 \mathrm{D})+\mathrm{H}_{2} \mathrm{O} \rightarrow 2 \mathrm{OH} \\
& \mathrm{e}^{-}+\mathrm{H}_{2} \mathrm{O} \rightarrow \mathrm{OH}+\mathrm{H}+\mathrm{e}^{-}
\end{aligned}
$$

\section{Diagnostics of reactive $\mathrm{NO}$ and $\mathrm{NO}_{2}$ species in the plasma gas}

For the diagnostics of the other important radical species such as $\mathrm{NO}$ and $\mathrm{NO}_{2}$, many scientists have generally used cavity-enhanced absorption spectroscopy (CEAS) [115] and Fourier Transform Infrared (FTIR) spectroscopy methods [116]. FTIR is a very useful radical analyzer based on absorption spectroscopy, also the CEAS has been employed in parallel with FTIR [116] for measurement of radical species by including the multipath cavity cell with a high reflection mirror [117] since the FTIR has a low sensitivity. CEAS has a higher sensitive absorbance $10^{-7} \sim 10^{-9}$, as compared with $10^{-3} \sim 10^{-5}$ in FTIR. Also, CEAS has a higher selectivity and a faster response time $[118,119]$ to be well adapted for the high-speed dynamics of RONS in plasma gas. Hence CEAS is suitable for in situ online analysis and real-time detection [118]. It is the most widely applied in various narrowband and broadband variants of cavity ring-down spectroscopy [116]. Also, it can be used in applications for the absorption measurement of liquid samples in a cavity containing a strongly absorbing medium [116]. For nitrogen dioxide $\left(\mathrm{NO}_{2}\right)$ measurement, the visible broad band cavity enhanced absorption spectroscopic (BBCEAS) method could be 
used, however, for nitric oxide (NO), CEAS should be used with a mid-infrared laser for diagnostics of infrared-active molecules, whose spectral range is between 3 and $20 \mu \mathrm{m}$ [120]. The $\mathrm{NO}_{2}$ absorption could be detected with ultraviolet and visible light sources such as LED and arc lamps. The visible absorption band of $\mathrm{NO}_{2}$ corresponds to the electronic transition in the molecule [121]. However, in the case of $\mathrm{NO}$, the vibronic absorption band of these species was located around $5.26 \mu \mathrm{m}$ and the absorption profile can be detected with a quantum cascade laser (QCL) [122]. This QCL, which can be adjustable to a specific wavelength of the laser, was widely used for $\mathrm{NO}$ absorption measurement. QCLs have the most suitable characteristics for absorption spectroscopy. For these reasons, $\mathrm{NO}_{2}$ and $\mathrm{NO}$ measurements could be carried out by BBCEAS and QCL-CEAS techniques, respectively. In this review, we report the measurement of $\mathrm{NO}_{2}$ and $\mathrm{NO}$ generated by a nonthermal air plasma jet using BBCEAS with lightemitting diode (LED) and CEAS with QCL, respectively. For the measurement of $\mathrm{NO}_{2}$ and NO density, a visible LED whose wavelength is $660 \mathrm{~nm}$ and a mid-infrared laser diode whose wavelength is $5.2386 \mu \mathrm{m}$ would be used, respectively. Radical densities can be calculated with the transmission ratio using Beer-Lambert law, which is obtained by the absorbed amount of laser intensity passed through the gas from the plasma jet in an optical cavity of CEAS [115]. QCL-CEAS was generally used to measure the absorption of $\mathrm{NO}$ radical species. Especially, the $\mathrm{NO}_{2}$ density has a value of $\sim 2.5 \times 10^{16} \mathrm{~cm}^{-3}$ in our air plasma jet. The NO density has a value of $\sim 4 \times 10^{15} \mathrm{~cm}^{-3}$ [115].

\section{Utilization of plasma bioscience for living health}

The healing mechanism of lesion tissues related to bacteria-infected diseases and virus-infected living tissues under plasma treatment could be clarified through basic and clinical researches by using food-born and air-born pathogens. Also, it is important to implement plasma medical devices for these kinds of medical purposes. This requires an absolute cooperation system between the two organizations for international standards of food safety and plasma medical devices, joint use of advanced research facilities, joint acquisition of intellectual property rights that will lead the world, and synergy of securing advanced technologies. In particular, the plasma bioscience field shows promising features that could be widely used in the prevention and treatment of COVID19, which is widely popular around the world.

\subsection{Cancer treatment application}

Ionizing radiation and chemotherapy both treatments have severe systemic side effects, and many cancers including brain, breast, lung, and gut cancers are notoriously resistant to these therapies. Failure of the immune system of the human body to detect and reject uncontrolled cells may lead to tumor or cancer development. Cancers use multiple mechanisms to escape from immune-mediated rejection. Several of these mechanisms are now identified on a cellular or molecular level. Plasma technology can be used as a therapeutic approach for treating these dreadful resistant cancers by modulating immune cells and tumor microenvironment. It can be used to cure intractable cancer lesions through changes in DNA, apoptosis, and cell cycle control proteins through the interaction of NBP or nanoparticles with them. Figure 16 shows the results of simultaneous treatment of Silymarin nanoemulsion (SN) and phosphate-buffered saline (PBS) treated with air DBD-NBP in xenografted mice with G361 skin cancer cells. Reactive oxygen radicals in cancer cells increase by about 3 times compared to cells without plasma treatment, and active nitrogen increases by about 2.5 times, which damages the DNA of cancer cells and reduces the size of cancer [29].

In addition, breast cancer cells that have recently developed resistance to anticancer drugs are an epiimmune mechanism by the active oxygen of DBD-NBP, and the sensitivity of anticancer drugs has been stably restored, and anticancer drugs can be used continuously. In
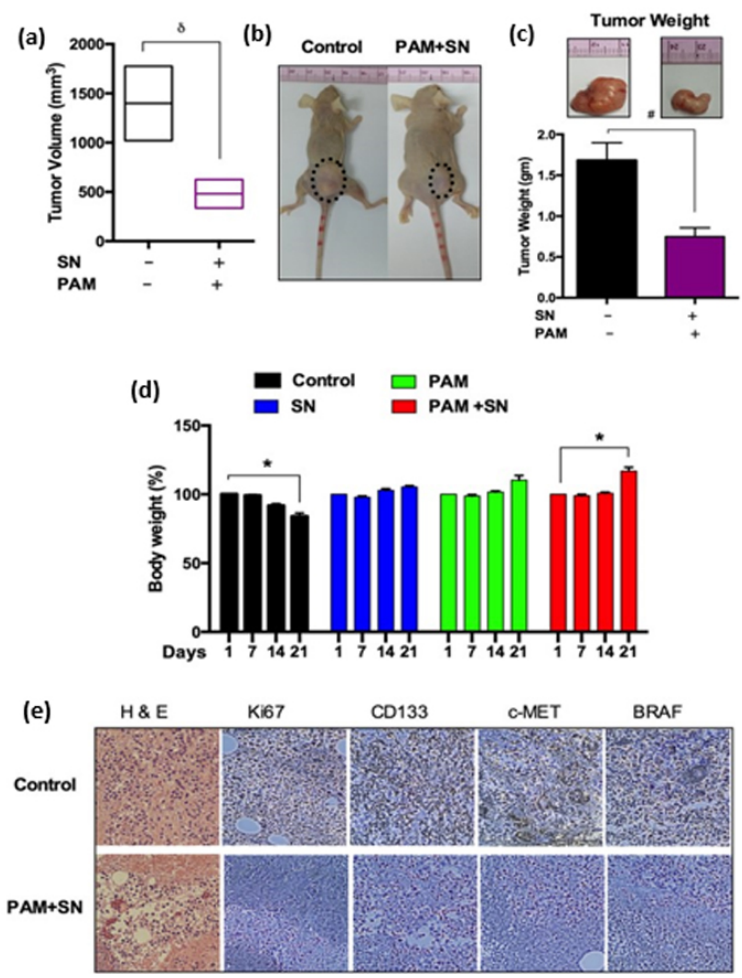

Figure 16. Anti-tumor effect of Silymarin nanoemulsion and plasma-activated PBS treatment in mice model. (a) Changes of the tumor volume in xenograft mice models. (b) Macroscopic observation of a control group and dual treatment of NBP-activated PBS and SN to nude mice group bearing subcutaneous tumors on the right hind flank. (c,d) Tumor sizes and body weight. (e) Immunohistochemical analysis of in treated and untreated tumor tissues as labeled. Reproduced with permission from [29], Copyright 2019, Springer Nature.

addition, plasma itself can target cancer tissues by restoring and promoting the immune system [123]. Current plasma medicine research mainly focuses on the non-thermal effects of plasmas. Several types of nonthermal plasma offer the ability to deliver reactive oxygen or nitrogen species indirectly or directly into biological tissues, indicating its possibility as an innovative tool for use in cancer therapy by branched organ or endoscopic targeting treatment technology. Also, plasma medicine researchers intend to establish a cancer-targeted approach by using non-thermal plasmas that will stimulate tumor inhibitory immune cells from their cancer-promoting state. As the community will learn more about the complexities of the proposed novel treatment method, they can be incorporate the safest and economic therapeutics into their strategy for innovative medical applications.

\section{Increasing cancer radio- and chemo-sensitivity}

In advanced stages, radiotherapy is a major treatment method for invasive and malignant tumors, nevertheless side-effects to normal counterparts and developed cancer cell resistance ease the effectiveness of radiotherapy. Since plasma technology has been shown to kill cancer cells successfully, Lin et al. [124] attempted an approach to combine plasma treatment with irradiation to improve radiotherapy. They demonstrated that plasma inhibited proliferation and induced apoptosis in many tumor cell lines being more lethal towards cancer cells over normal cells. These promising synergies of plasma provide sensitivity to radio-resistant cancer cells by producing ROS, inducing apoptosis, and cell cycle arrest. Besides radio-sensitization, plasma has been shown to improve cancer cell death in glioblastoma cells which are resistant to temozolomide (TMZ). One group of researchers evaluated the anti-cancer properties of plasma at different dosages both in TMZ-resistant and TMZ-sensitive cells and demonstrated that co- 
treatment of plasma and TMZ led to induction of cell cycle arrest resulting in the inhibition of cell growth, thus plasma might act as a promising candidate to restore the sensitivity of resistant glioblastoma cells regarding TMZ therapy [125].

On the other hand, scientists also tried to work on improving plasma efficacy towards selective and preferential cancer cell killing. In line, Kaushik et al. [21] showed the selective and efficient anti-cancer effects of plasma at a low dose can be achieved when cell metabolic modifiers; 2-Deoxy D-glucose (2-DG) are used together. Their findings suggest that incorporation of 2-DG boosts the efficacy of plasma by targeting apoptosis with caspase-dependent and independent pathways and glycolysis for cancer cell death inhibition. This methodology is the first report to show the advantage of combining plasma with 2-DG to eradicate blood cancer cells [21]. Additional studies were performed to examine the combined treatment of plasma and SN on melanoma cells in vitro and in vivo. Notably, these dual-treatment strategy effects on the epithelial-mesenchymal transition (EMT) and cancer stem cell maintenance. Co-treatment of SN and plasma-activated medium showed good potential on reduction of tumor growth and mice body weight and melanoma-markers as seen in tumor xenograft nude mice model [29].

\section{Immuno-activation and immunogenic cell death}

In immunogenic cell death (ICD), characterization, and identification of various stimuli that induce these types of cancer cell death has remarkable potential to develop the effects of cancer therapy. Using in vitro colorectal cancer cells, one study showed that plasma treatment stimulated the release of dangerous signals responsible for ICD. Moreover, they showed that protective immunity in the CT26 tumor mouse model can be achieved when treated cells were used as a whole-cell vaccine as seen by elicited danger signals and recruitment of antigenpresenting cells into subcutaneous tumors. This study highlights the first confirmation that the plasma could have potential for cancer immunotherapy and can be applied to clinical translation [22].

The toxicity findings showed that the treatment of HeLa cells with 4 min of DBD plasma and $7 \mu \mathrm{M}$ of NO-PAW could reach almost $\mathrm{IC}_{60}$. For the apoptosis method, 4 min treatment of DBD plasma could in duce $7 \%$ apoptotic effect, whereas $7 \mu \mathrm{M}$ NO-PAW could induce $18 \%$ apoptotic effect. In addition, this research assumed that DBD plasma and NO-PAW can induce HeLa cell apoptosis by facilitating an accumulation of intracellular RONS. RNS, including NO* has been known as one of the main regulatory molecules in immune biology.

Plasma-synthesized environmental friendly mussel-inspired gold nanoparticles induces apoptosis and autophagy-mediated damage-associated molecular pattern release to stimulate immunogenic cancer cell death. Moreover, these plasma systheiszed nanoparticles showed enhanced uptake in cancer cells and less toxicity of non-malignant cells [126].

Recently, Kaushik et al. [127] reported that plasma treatment stimulates monocytes and macrophages thereby inhibiting the growth of tumor cells in the co-culture system via TNF- $\alpha$ and iNOS release. Moreover, the plasma does not affect immune cell viability as seen in macrophages. These results indicate that reactive species induced by plasma could recruit or activate cytotoxic macrophages to cancer cell death induction [127]. In another study, they discovered that plasma could differentiate pro-inflammatory macrophages from monocytes which favors anti-cancer immune responses in aggressive resistant cancers [31]. This study uncovers that the use of plasma technology could be beneficial in modulating the pro-tumor microenvironment via affecting resting immune cells.

Targeted cancer therapy by plasma using nanotechnology-based approaches

Tremendous efforts have been conducted over the last few years aiming on the utilization of synergistic impacts of nanomaterials and plasma for cancer applications. The combination of these two emergent research areas typically belongs to two different strategies. One of them is the synthesis of nanomaterials using plasma, which has been evidenced as a quick, simple, cost-effective, and effective method with eliminating the utilization of toxic chemicals during the fabrication process of nanomaterials, typically used in conventional techniques. Therefore, the plasma-synthesized nanomaterials are comparatively less toxic which makes them more suitable for practical applications in the biomedical field. The other one is the combination of plasma synthesized nanomaterials along with plasma. This strategy relies on the fact that the cell membranes can be open or enlarged upon plasma treatment, thereby enhancing the cell permeation. Subsequently, nanomaterials can easily enter or penetrate through cellular membranes.

Additionally, the selectivity of plasma can be balanced by functionalized nanomaterials, which have specific targets [32]. This concept allows us to study enhancers of plasma activity using nanomaterials in plasma medicine. Since plasma treatment did not provide any selectivity among cancer and normal cells. To overcome this issue, Kim et al used an antibody-targeted specific tumor protein and gold nanoparticles (GNPs) to synthesize functionalized GNPs to enhanced the anti-melanoma effect [128]. When this cancer-specific antibody conjugated GNPs was pretreated before plasma irradiation, these functional GNPs binds selectively to target cancer cells, which are then efficiently sensitized by the plasma resulted in a reduction in the growth of cancer cells. This technique shows the probability to enhance plasma therapy selectivity [129]. Another group of researchers has shown the effects of co-treatments that included polyethylene glycol-coated GNP and plasma on the tumor relapse phenomenon (maintenance of cancer stem cells and EMT) which have not been much described so far. They suggested that their combination inhibited the proliferation in cancer cells by inactivation of the PI3K/AKT signaling axis. The reversal of EMT by GNP and plasma also delays tumor growth in tumor xenograft mice models by decreasing mesenchymal markers [33].

In another study, they observed that enhanced anti-cancer effect in glioblastoma cells was achieved by gold quantum dots and plasma co-treatment. These co-treatments can induce cancer cell apoptosis through Fas, TNFR1, DR5, and DR4 receptor-mediated extrinsic pathways. These studies also provide evidence that improvement of cellcell contact adhesion was observed by gold quantum dots attributed to target drug delivery systems using plasma [34]. Owning the fruitful approach of plasma towards nanoparticle synthesis, Linh et al. [130] developed an exceptional single-step synthesis protocol using a plasma jet to simplify the fabrication process of polydopamine-functionalized gold nanoparticles (PDA-GNPs), eradicating the use of additional toxic chemicals.

Plasma treatment shortened the reaction time, required for PDAGNPs compared to conventional chemical methods. Specifically, they showed that quickly fabricated have high cellular uptake and cytotoxicity against breast cancer cells which underline the importance of plasma-synthesized PDA-GNPs for declining cancer cell growth through their intracellular delivery [130].

A comparison study on the photodynamic anticancer activity of magnetic particles was carried out [131], by synthesizing multifunctional magnetic particles and using two different ferrite sub-micrometer particles $\left(\mathrm{Fe}_{3} \mathrm{O}_{4} @ \mathrm{HP}\right.$ and $\left.\mathrm{CoFe}_{2} \mathrm{O}_{4} @ \mathrm{HP}\right)$ with different surface properties. Two different multifunctional particles had the same particle sizes within the error tolerance of $4.5 \%$. Biocompatibilities and photodynamic anticancer activities on mammalian cells were evaluated, finding that both have excellent biocompatibilities without cytotoxicity and having anticancer activities in the concentration range of $0-50 \mu \mathrm{g} / \mathrm{mL}$. Multifunctional cobalt ferrite $\left(\mathrm{CoFe}_{2} \mathrm{O}_{4}\right)$ nanoparticles $\left(\mathrm{CoFe}_{2} \mathrm{O}_{4}\right.$-HPs-FAs) functionalized by coating them with hematoporphyrin for establishing photo-functionality and by conjugating with folic acid (FA) for targeting cancer cell were synthesized [132].

The activities of the $\mathrm{CoFe}_{2} \mathrm{O}_{4}$-HPs-FAs were evaluated by inves- 


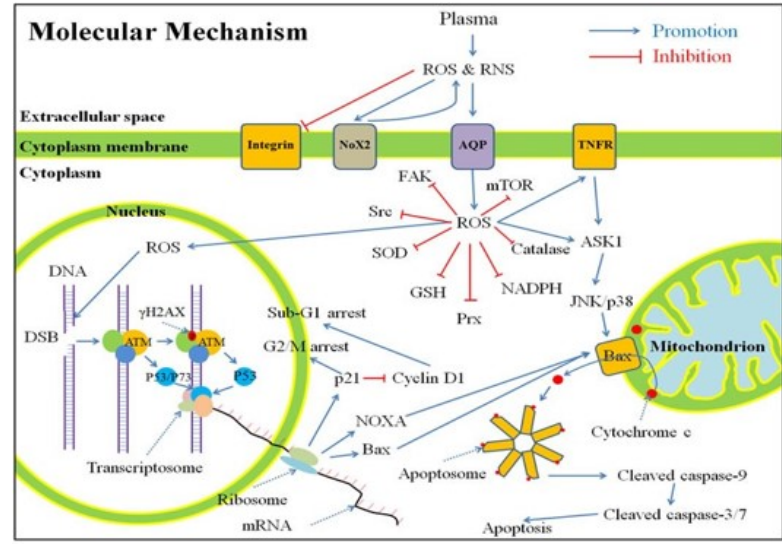

Figure 17. The summary of the anti-cancer mechanism of NBP. Shortly, the NBP generated reactive species will trigger an evident rise of intracellular ROS, which diminishes the intracellular anti-oxidant approach and also causes DNA doublestrand break. Consequently, cell cycle arrest and apoptosis based on tumor necrosis factor receptor or mitochondrion-pathway occur. Reproduced with permission from [133], Copyright 2017, Impact Journals.

tigating magnetic resonance imaging (MRI) in vitro, photodynamic anticancer activities, and its biocompatibility, on FA receptor (FR)positive and FR-negative cell lines, Hela, KB, MCF-7, and PC-3, to use for medical applications. This assessment study showed that the $\mathrm{CoFe}_{2} \mathrm{O}_{4}$-HPs-FAs have good biocompatibility and MRI application with non-cytotoxicity, and significant photodynamic anticancer activities at low concentrations irrespective of cell types. These studies indicate that nanoparticles stimulate the functional loss of specific cellular proteins or signaling pathways, which oppose the intracellular variation due to the plasma-generated active species.

Molecular mechanisms involved in plasma-induced anti-cancer effects

Irrespective of whether the plasma-induced effects are indirect or direct, the proper irradiation time should be examined. It has been observed when the exposure time is very long, both cancer and normal cells are destroyed by plasma, while in case of short exposure time, neither cancer nor normal cells are destroyed. Therefore, optimization of the therapeutic range of plasma activated media (PAM) and plasma is crucial. As discussed above, sensitivities of PAM and plasma are different depending upon cell types.

Although many scientists attempted to discover the intracellular molecular mechanisms involved in selective cancer cell killing so far, however, there is not clear strong evidence to date. Plasma interacts directly with not only cancer cells but also with the liquids surrounding cancer cells. Differential studies proved that reactive species are the main culprits for plasma-induced cytotoxic effects; though, other mechanisms have been also recommended. In the forthcoming time, details regarding plasma-induced effects on gene regulation, cellular signaling, and metabolic networks will be clarified in detail for clear understanding (Fig. 17).

Hou et al. [134] discovered that the plasma treatment could activation of p53 and MAPK signaling pathways, ensuing in changes in different regulatory gene expression patterns. Their tumor transcriptome analysis helped to recognize the key players in modulated gene expression following plasma exposure at the molecular level, which also aided the interpretation of the downstream pathways. These studies build the foundation for additional studies to more clarify the functions of numerous pathways responsible for plasma-induced biological processes [134].

Another attempt was done by Tanaka et al. [135] by investigating the differences in intracellular molecular mechanisms among different plasma-treated solutions using brain cancer cells. Intriguingly, PAM has been shown to induce oxidative stress-dependent, while plasmatreated RL solutions induced oxidative stress independent cancer cell death. They concluded that different plasma-activated solutions could be used for the various cancers that are resistant to particular plasmatreated liquids in the future [135].

\section{Targeting tumor micro-environmental for plasma-induced} anti-cancer effect

Recently, plasma exposure is widely considered as a local therapy, it can also modify the cell-to-cell mechanisms and cell-to-extracellular matrix (ECM) communication in distant tumor sites. Nonetheless, it has remained undiscovered how the physical interactions take place among cells and the ECM in the tumor microenvironment that affect the outcomes of plasma therapy. The tumor microenvironment (TME) consists of non-transformed and malignant cancerous cells, tumor vasculature together with ECM.

Dynamically, non-malignant cells actively participate in the TME throughout carcinogenesis phases where their tumor-promoting function is reflected [136]. Particularly, it is crucially important to know how cells respond to the signals induced by plasma-generated reactive species and further how could plasma affect these cellular mechanisms. Currently, there is an increased number of evidence using complex 3D cell culture models, in combination with other cells or alone of the TME. These technologies provide great advantages to mimic the response to treatment acquired in real complex solid tumors, such as the determination of the effect of plasma on immune cells, ECM components, stromal cells, secretion factors, and so on. Likewise, it is possible to use these technologies probably for clinical plasma application in patients. Understanding how plasma influences the mechanism of communication between TME and cancer cells is of outermost relevance to apply cancer plasma treatments which can be translated into the clinic in the future [137].

\section{Clinical trials and highlight growing demands for cancer application}

In the plasma community, plasma oncology is a growing scientific field with uncounted important open questions. These answers will be essential for the further development to a thoughtful medical treatment of cancer using plasma for clinical purposes. Isbary et al. suggested that different plasma devices should be standardized for their capacities and the resulting plasma chemistry in the liquid phase would be beneficial to compare different approaches [35]. First animal studies have been performed on subcutaneously induced solid tumors using transcutaneous plasma treatment [138], which agrees on the general perception of plasma-induced anti-cancer treatment [25]. Besides, local plasma treatment induced effective inactivation of single layers of cancer cells [139], supports the use of plasma combining with surgical treatments, where large-sized tumor removal is impossible.

Von Woedtke et al. successfully proved that plasma eliminates remaining cancer cells following post-surgical treatment [140]. These reports suggest that the next important step should be to upgrade plasma devices including real-time and continuous monitoring of both plasma functioning and the target that is treated. In line, a very promising approach was shown with the use of plasma-treated solutions for $a b-$ dominal lavage in the case of disseminated tumors [141], where both radiation and surgical treatments were impossible and chemotherapeutic drugs also cause severe side effects. Another promising therapeutic approach of the use of plasma-treated solutions was confirmed through animal studies $[142,143]$. Though, much more research is still required before starting a clinical application.

\subsection{NBP applications to dental sciences}

In dental science, reactive species can be found all treatment procedures such as in laser treatment, bleaching chemicals, cold plasma, 
photodynamic therapy using photosensitizers, and even in resin cement, all of these strategies contribute to the generation of reactive species. Some of these technologies may have negative effects on cells due to the high amount of by-products and reactive species from composites bleaching agents and some technologies such as NBP have a positive effect on dental cells. The NBP has been used as a source of ROS/RNS for various medical applications and also has the potential for direct dental and dental stem cells based applications. Reactive species play crucial roles in intracellular redox reactions and signaling pathways. At minimal or moderate doses, they can be extremely advantageous for biological processes such as angiogenesis and repair, whereas at extremely elevated concentrations, can induce tissue injury or cell death, or apoptosis. Non-thermal plasma-generated reactive species can be utilized to eliminate periodontal pathogens and related diseases. Currently, the researcher only focused on periodontal ligament stem cells however therapeutic application on other types of dental stem cells remains largely untapped. Up to now, little is known about the effect of NBP on mesenchymal and dental stem cells [144], and understanding the therapeutic outcomes of reactive oxygen and nitrogen species is one of the main focuses of the investigation for prospects of this NBP technology with different parameters tuning [144]. Attempts must be made to expand the study on the interaction of reactive species with dental stem cells for more speedy tooth bone formation and dental wound healing. More innovative ideas on the use of plasma in the field of dental research are needed so that effective noninvasive treatments can be given for oral diseases.

NBP can be used to sterilize dental medical equipment which can be an alternative to traditional sterilization methods with a high level of safety and low cost [145]. Researchers also studied the influence of direct or indirect cold plasma on the various microbial cultures. It has been indicated that NBP induces a greater effect to gram-negative than the gram-positive culture [146]. The World Health Organization (WHO) believes periodontal infections and dental caries as a key cause for tooth loss and a worldwide health issue [147]. The main culprits for this are the infection-causing microbes (i.e. pathogens) which need to be thoroughly removed. Therefore, the use of mouthwashes is very important for good oral health. Most commonly, commercial or market available mouthwashes are used but those can trigger tooth staining and erosion, hence better options need to be investigated. In vitro study showed by Li et al. indicated the anti-bacterial effects of NPB activated water as a mouthwash [148]. It is proved that Streptococcus mutans, Actinomyces viscosus, and Porphyromonas gingivalis are generally responsible for dental carries, plaque formation, and chronic periodontitis. NBP treated water can be obtained by exposure of distilled or mineral or tap water to plasma discharges safely to generate reactive species such as $\mathrm{NO}_{\mathrm{x}}$ and $\mathrm{OH}$. The NBP treated water successfully inactivates (damage to proteins, genetic material, morphology disruption, and damage to cytoplasm) microbial pathogens responsible for oral diseases. The mechanism of inactivation is based on NBP generated reactive oxygen and nitrogen species (such as $\mathrm{NO}_{\mathrm{x}}$ and $\mathrm{H}_{2} \mathrm{O}_{2}$ ). These species may have been capable to reduce microbial growth and infectivity potential exponentially. Also, the NBP treated water or media for dental application undergoing a lot of research. One of the main applications is for tooth whitening procedures as well although only a few products are available in the market for usage. Up to now, only tooth bleaching method is used for tooth whitening, which can be toxic to other normal dental tissues or cells after long use. While based on efficiency, safety, and biosecurity the application of NBP or NBP treated water or solutions can be potential methods for tooth whitening. Recently Kang et al. analyzed the bleaching efficiency of NBP treated solution with reactive species capable to increase the oxidation-reduction potential [149]. Other studies also supported this claim for the utilization of this technique $[149,150]$.

Surface modification is another focused research area in the dental sciences. Nowadays interest in tissue bioengineering is ever increasing due to several reasons that include restoring and replacing the dam-

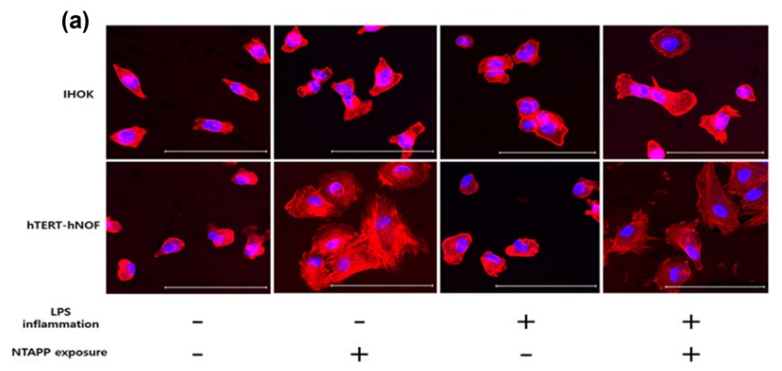

(b)
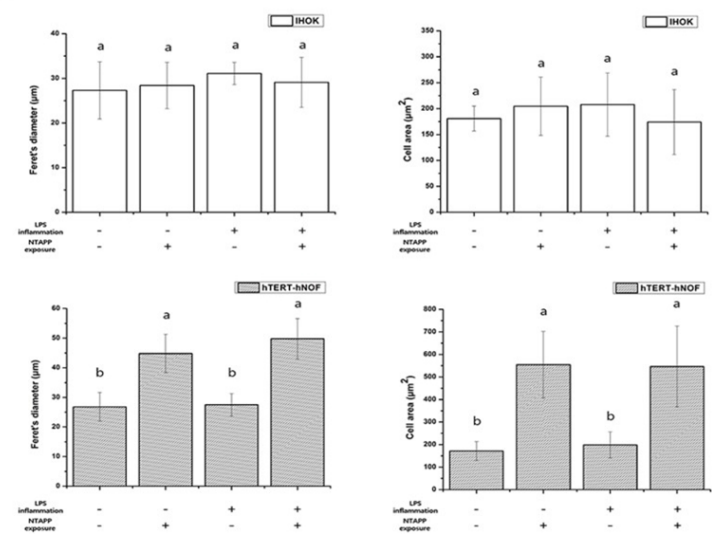

Figure 18. Behaviors of nonthermal biocompatible plasma treated titanium surface on oral soft tissue cells. Reproduced with permission from [159], Copyright 2018, Springer Nature.

aged or non-functional organ or tissue. There have been numerous efforts to enhance tissue rejuvenation in dental science by several approaches. Recent researchers highlight activating dental cells by surface modification of either biomaterial or extracellular matrix. However, the complete mechanism on dental cell activity alteration and antimicrobial efficiency of this modification is still not reported yet. Also, these modifications on biomaterial or biological tissue-relevant surfaces are not an easy task due to several limitations such as damage of biomaterials and loss of biocompatibility after treatment. NBP can be applied to sterilized biomaterials with surface functionalization. NBP treatments are safe and cost-effective and have many advantages compared to conventional methods [151].

NBP secures great capabilities such as for bio-sterilization, bacterial and virus inactivation, and dental caries treatment [152-154]. NBP can be used to dental cosmetic-related problems such as gummy smiles and gingival hyperpigmentation as well as soft tissue treatments [155]. Also, NBP as the source of RONS can be used for dental stem cell applications for more speedy bone formation and dental tissue healing together with molecular expression linked to growth factors $[144,156]$. NBP treatments can have an impending application on augmentation of dentin adhesion [157]. NBP exposure for less than a minute increases adhesive properties of biomaterials by enhancing nano hardness, young's modulus, and hydrophilicity of various materials [158]. Earlier investigation indicated that NBP treatment improved surface biocompatibility of implants on titanium (Fig. 18), zirconia, and polyetheretherketone surfaces.

Surface modification of dental biomaterials using NBP has gained major demand in the last few years. Various plasma-based surface modification methods such as spray, polymerization, and plasma immersion ion methods are well investigated and are used in the biomedical industry [160]. These NBP based biomaterials surface modification methods have great potential due to economic and efficiency reasons. Zirconia and Titanium are the main materials that have been 


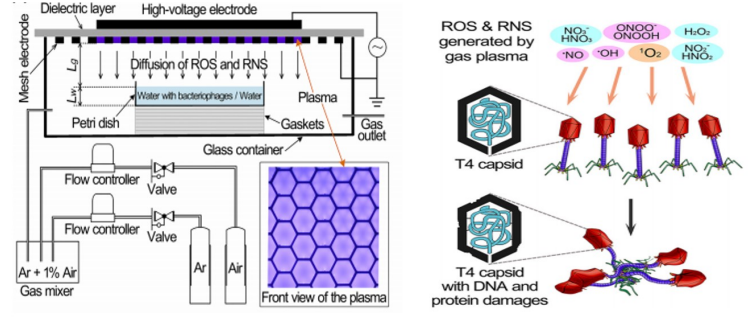

Figure 19. RONS generated by surface plasma for virus inactivation. The inactivation of bacteriophage T4 by ROS and RNS of plasma. Reproduced with permission from [164], Copyright 2018, ASM Journals.

utilized for a long time in orthopedics and suitable dental materials field for implants. The nature of these materials is biological inert, so can be important for inflammation or adverse immune reaction. However, these materials can not support any bone formation or growth of dental tissue. NBP based biofunctionalization or coating procedures can be used on these materials to improve bone formation or tissue growth capabilities. Yeo et al. proved that plasma coating of a carbon-based plasma polymer layer on zirconium biomaterial enhanced biofunctionalization with increased bone cell response [161]. It is also investigated NBP induced surface wettability with the antiadhesion effect of bacterial strains on hydrophobic materials such as poly-dimethyl siloxane (biomaterial). Another investigation showed that found that NBP treatment on titanium dental implant biomaterials surface with air gas can support soft tissue integration with controlled cytokine release [159]. This kind of biomaterial-based advantageous effect may provide the basis for new strategies. There are several possibilities using NBP treatment in the field of dental research areas. Therefore, the possibilities of utilizing such NBP technology for dental treatment is deemed, it would provide a new base for novel dental devices in the future and likely to have a global impact. New concepts in this area can further be utilized for future sterilization, stem cell growth, and differentiation, tooth whitening, enhanced surface modification of dental biomaterials using NBP.

\subsection{Plasma application to the COVID19 (SARS-COV2: Coronavirus 19)}

SARS-COV2, or COVID19, originated in Wuhan, China at the end of 2019, has now spread worldwide at an alarming rate, infecting about 100 million people, and causing about 2.3 million deaths, by an approximate death rate of $2.2 \%$. In addition, confirmed cases continue to spread and become infected in the US and Europe, and a vaccine against it has now been under development for injection and some countries are now vaccinating for prevention. Viruses occupy the largest number in the past billions of years on the earth [162], and various types of them exist and have adapted, evolved, and mutated according to the environment of the times.

Viruses play an important role in the global ecological environment, such as the carbon cycle of the sea [163]. On the other hand, pathogenic viruses infect tens of millions to hundreds of millions of animals each year on the planet or infect people. This has resulted in a decrease in crop yield or the loss of many lives. Therefore, the inactivation of harmful viruses is an essential and very important issue for human health. The inactivation of the virus can be successfully processed using reactive oxygen and reactive nitrogen in plasma [164]. Figure 19 (left side) shows the surface discharge NBP consisting of a mixture gas not only a large number of electrons and ions, but also various reactive oxygens and various nitrogen species at room temperature, that is, a cocktail, so it can inactivate bacteriophage T4 through their complex action. The cocktail interaction of these species brings damages to the DNA and protein of the bacterial phage as shown in Fig. 19 (right side). Corona 19 virus and its strains, which are highly infectious, can also be sufficiently inactivated by environmentally friendly application of NBP plasma, which is considered a new hope for humanity [165]. Their research field is relatively young and short, and started about 15 years ago, almost coincident with the viewpoint of plasma bioscience [166-168]. At that time, it was known that ozone inactivates the virus. Due to environmental safety standards, many researchers are conducting their investigations and development on the inactivation of viruses using other eco-friendly reactive gases while keeping ozone below that. Cold plasma, green or environmentally friendly technology has been widely used in many areas for microbial sterilization or inactivation. Recently, plasma medicine researchers initiated preliminary studies on SARS-COV2 using cold plasma devices [165, 169-178]. Both, plasma and plasma-activated water treatment showed inactivation of viruses in a dose-dependent manner, and additionally in vivo investigations indicated no side-effects with low invasiveness, simplicity, improved wound healing, inhibition of pain and inflammation $[165,177]$. Several studies have reported the influence of cold plasma treatment on bacteriophages (T4, $\Phi 174$, and MS2 strains) and viruses such as Newcastle disease virus (NDV), human norovirus (NV), porcine reproductive and respiratory syndrome (PPRS), Feline calicivirus (FCV), murine norovirus (MNV) and hepatitis A virus (HAV), Respiratory syncytial virus (RSV), etc. Plasma generated RONS, electrons, ions, ozone, and other physical factors play that can affect structural components (cell walls or cell membranes) as well as physiological components (nuclear material and proteins). However, concrete proof on inactivation of human virus pathogenesis and alteration of host-pathogen interaction mechanism is not reported yet. There is a growing need to investigate plasma-based virus inactivation mechanisms in both cell-based models and in animal models (including organoids) against future coronaviruses. The mechanism for the inactivation of coronaviruses will be the targeting virulence factor and host-pathogen interaction using nonthermal plasma that may give new insight for the control of virus pathogenesis (Fig. 20).

Also, AI-based prediction models can be used to predict possible mutations based on existing data or the next mutated virus sequence. These models can help early prediction of virulence properties which can help in preventing infection and support in NBP based treatments. The development of economical and cost-effective NBP based treatment methods will provide endless opportunities for critical stage patients infected with the corona virus. Utilization of the NBP based virulence factor and host-pathogen interaction control strategies will address challenges raised by virus pathogenesis and will promote the development of novel plasma-based treatment methods, that can show an encouraging potential for clinical use. In addition, in the reality that research on the interaction between plasma and virus is recognized to be important to clarify how to inactivate COVID19 and its variant virus in the development of plasma quarantine devices for human beings. Furthermore, it is time to conduct research and development ensuring safety to patients and medical doctors in the implementation of plasma treatment devices for active treatment of COVID19 and its variant virus around the world. In addition, the simultaneous development of plasma-treated water for this needs to be actively considered for living and health, and the justification for this is overflowing, as discussed.

\subsection{Plasma for disc herniation treatment}

Lumbosacral herniated nucleus pulposus (HNP) mainly responsible for low back and lower extremity pain symptoms of lumbosacral herniated nucleus pulposus, and they are attributed to damaged annulus inflammation and neuronal impingement of herniated disc tissue. Also, lumbar spinal pain leads to impaired movement and reduced involvement in activities. Certainly, a disc-related disorder in the industrialized population is incredibly frequent and causing restrictions and work disruptions lead to enhanced costs for clinical treatment and reduced productivity. Taking into fact, the most favorable treatment 


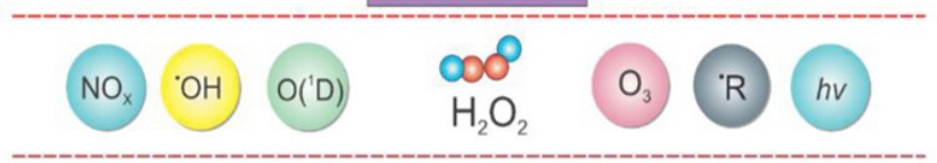

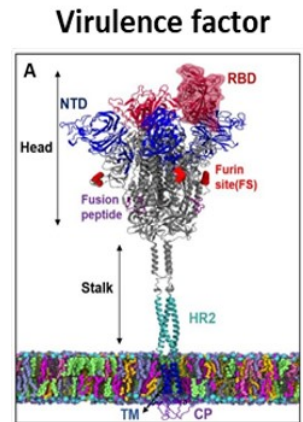

(e.g. Spike protein )
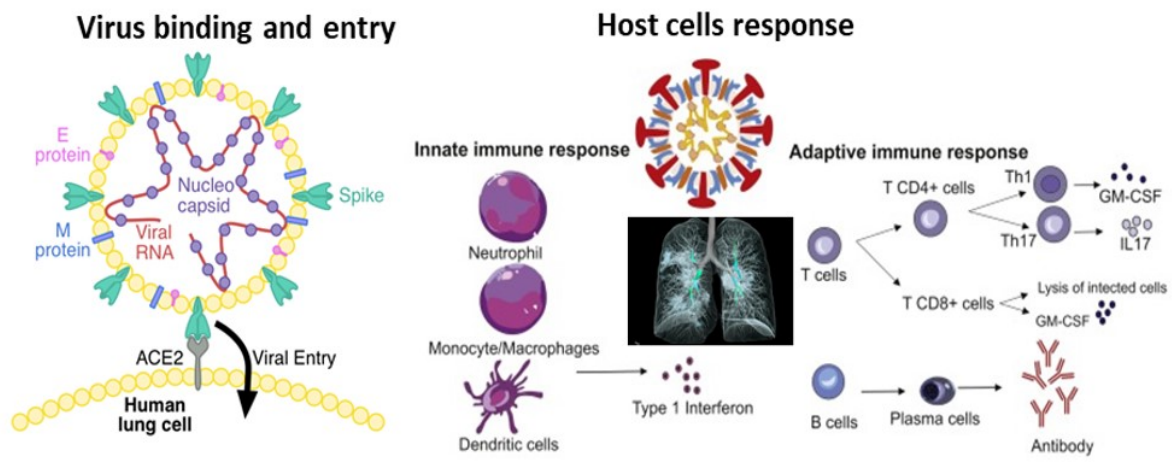

Oxidation/attenuation

- Functional impairment
Binding incompetency

- Entry blockade
- Immunomodulation

- Lung rejuvenation

- Pulmonary vasodilation

Figure 20. Possible mechanism on corona virus inactivation using NBP.

must comprise a short treatment time, quick healing effects, quick return to daily activities, and cost-efficient. Generally, open discectomy is suggested if conservative therapy fails. The success rate of the discectomy procedure is stated to be between 70 to $90 \%$. However, there are several issues in this surgical procedure such as moderate or severe neuronal damage, undistinguishable response to the oral painkillers, as well as the risk of relapse.

Recently, to minimize disadvantages several minimally invasive spine intervention procedures and sources are being established to augment the surgical improvements. Minimally invasive interventional methods and tools for tissue ablation using catheter insertion have been shown to reduce local pressure and cauterize irregularly growing nerve endings. Recently Kim et al. also created a radiofrequency plasmabased catheterization procedure and investigated its clinical outcomes [178]. They invented a navigable plasma disc decompression catheter using a radiofrequency ablation technique that was devised for local access and selective HNP treatment. Clinical efficacy of this device in patients with disc herniation with selective targeting, safe removal of localized tissue through ablation. The radiofrequency-based focal removal procedure using a maneuverable plasma disc decompression catheter is an optimized and feasible option for the treatment of lumbar disc herniation with minimum invasiveness [178]. In the future, critical attention must be given to the probability of electrical damage to neuronal tissues. Therefore, additional technological improvements are needed to increase the effectiveness of tissue ablation and safety standardization issues.

For the development of new plasma-based device for disc treatments, Hong et al. recently invented the Navigable Percutaneous Disk Decompression Device (L'DISQ) and navigable percutaneous plasma cervical decompressor (L'DISQ-C) (Fig. 21) [179]. These devices are based on a minimally invasive procedure for percutaneous disk decompression that utilizes plasma energy to damage unwanted nuclear tissue. Moreover, the investigation was performed to compare effects by L'DISQ and L'DISQ-C to treat disc herniation. It was reported that the cervical disc (L'DISQ-C) has comparable efficacy and safety as the lumbar device (L'DISQ). In conclusion, L'DISQ-C wand can be navi-

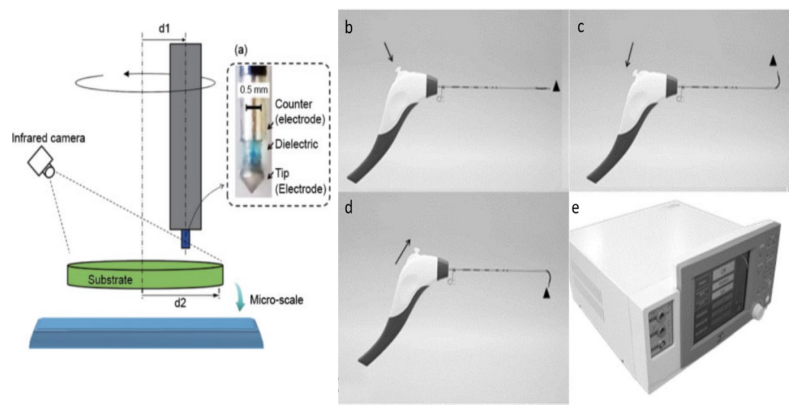

Figure 21. Experimental setup for aqua plasma properties and images of the L'DISQ-C. (a) Procedure tip of L'DISO (d1) The distance was $5 \mathrm{~cm}$ from the center of the electrode. (d2) the target substrate (Nucleus pulposus) for plasma ablation, was contained in a culture dish. (b-d) Images of the L'DISQ-C and (e) its power source. The tip of the wand can be curved to the preferred angle by (b) downward or (c) upward by the rotation of the control wheel. The power generator supplies $100 \mathrm{~W}$ electric power to the plasma-generating wand. Reproduced with permission from [179], Copyright 2018, Thieme, [180] Copyright 2019, Thieme.

gated into the posterior region of cervical disks and could ablate target disk tissues quantitatively without substantial structural or thermal to adjoining tissues. Lee et al. also evaluated safety by measuring the increase in temperature at various distances from the device tip within the nucleus pulposus. It is analyzed that denaturation of collagen and other bio-relevant molecules can not be affected during herniation treatment as temperature rise was less than $13^{\circ} \mathrm{C}$ (from 23 ${ }^{\circ} \mathrm{C}$ ) at all measured distances [179]. Therefore macroscopic damage could not have occurred in the neighboring tissues. The possibility of unknown factors is also emphasized in the plasma device which generates hydroxide, electron, ultraviolet, and other reactive species, and can affect at the microscopic level in the treatment area. Further investigation on the effect of this procedure on normal blood perfusion, body temperature, and other physiologic conditions in cells or tissues is needed. Future study on the animal model will be important to achieve a mechanism for the effects of the L'DISQ-C on cervical disk herniation. 
Another recent study described optimal parameters of aqua-plasma beams for intervertebral disk resection. In this investigation, a minimally invasive method for intervertebral disk resection in continuation of previous studies using plasma beams has been developed. Two main factors of plasma must be considered for herniated disc treatment in medical settings: the disc tissue ablation effectiveness and the safety standards of the method. Conventional parameters such as voltage and tip speed were analyzed based on clinicians' personal experiences and laboratory experiments. High-frequency plasma-based ablation is called coblation which is a nonthermal method for surgery. High-frequency energy is applied via the energized bipolar electrodes to a physiological medium (saline solution), producing a highly focused plasma field around the electrodes. These ionized particles have enough strength (3-6 eV) to break organic molecular bonds within the tissue. The ablation rate was assessed using porcine nucleus pulp at various procedural tip speeds and voltages. The voltage is directly proportional and tip speed is inversely proportional to the levels of plasma generation. The high amount of plasma formation is likely to enhance the efficiency of disk removal. However, a high amount of heat generation will be a drawback of this procedure for medical use irrespective of target removal. As per thermal safety generation of heat during the experiment is also analyzed. The higher ablation rate was obtained at slower procedural speeds and higher voltages. The optimal plasma parameters for minimal tissue damage were 280 volts rootmean-square $\left(\mathrm{V}_{\mathrm{rms}}\right)$ and a $2.5 \mathrm{~mm} / \mathrm{s}$ procedural tip speed. The plasma ablation procedure is distinct from other medical laser procedures or intradiscal electrothermal therapy (IDET) as it uses chemically reactive species such as hydroxyl radicals generated around the tip instead of heat energy to damage the disk molecular structure. These findings on disc treatments provide several useful pieces of information for safe and effective procedures in clinical settings. A further investigation is needed to show the impact of the speed and voltage in animal models or human patients.

\section{Perspectives and conclusion}

Plasma bioscience and medicine are newly developed and rapidly developing with the goal of next-generation health promotion and disease healing of a new concept. To this end, the development of a nonthermal (or low temperature) atmospheric pressure biocompatible plasma (NBP) source that can be applied to the actual medical field, and research and development of PAW using this NBP are actively underway. These NBP and PTW could also be applied to cancer cells, neurodegenerative diseases, viral diseases, and skin diseases that are difficult to treat. There is a need for active cooperative research such as securing new data and related basic plasma clinical trials. The treatment based on plasma bioscience can be combined with existing cancer treatment methods and can be applied in various ways. Also, recently researchers examined effect of plasma treated biomolecules for biological application [181]. This area also have potential for several clinical applications. In particular, NBP could be expected to have great effects when it can be applied with plasma-treated water together to liver cancer, lung cancer, brain cancer, breast cancer, pancreatic cancer, skin cancer, and blood cancer, which are known to be types of some intractable cancer. The manufacturing and disinfection of plasma medical devices for surgical trauma treatment wound healing as well as burn treatment, and skin disease control and treatment are recognized as very important fields. In addition, the efficiency of NBP can be expected when sterilizing bacteria resistant to antibiotics. Recent progress in plasma bioscience and medicine showed utilization of non-thermal gas plasma for bio-sterilization, water purification, and decontamination $[182,183]$. Also nonthermal gas plasma is used for agriculture applications [184] and this area can be further explored with several new ideas. Now, in the era of long-lived health and welfare, it is also important to secure cooperative technology to solve this by applying NBP to the degenerative brain and disc herniation diseases of the silver generation.

In 2015, we need to get a database of realistic wisdom about the valuable experience of Middle East Respiratory Syndrome (MERS) and the infection of a new virus COVID19 since last year, as seen in the harsh reality. Prevention and inactivation of these viruses in allweather areas, indoor disinfection of ambulances, indoor air purification and virus removal, water purification, and fine dust removal are difficult to implement only with conventional methods. At this time, applying NBP technology can bring better results than expected.

\section{Acknowledgement}

This study was supported by the National Research Foundation (NRF) of Korea, funded by the Korean government NRF2016K1A4A3914113 and 2021R1A6A1A03038785, and also partially supported by the Kwangwoon University in 2021.

\section{Conflicts of Interest}

The authors declare no conflicts of interest.

\section{ORCID}

Eun Ha Choi https://orcid.org/0000-0001-5385-1878

Young June Hong https://orcid.org/0000-0002-1831-8004

Nagendra Kumar Kaushik https://orcid.org/0000-0002-4965-5046

\section{References}

[1] E. H. Choi, Vacuum Magazine 7, 2 (2020).

[2] M. G. Kong, G. Kroesen, G. Morfill, T. Nosenko, T. Shimizu, J. V. Dijk, and J. L. Zimmermann, New J. Phys. 11, 115012 (2009).

[3] E. H. Choi, Y. H. Kim, and K. N. Kim, Vacuum Magazine 1, 38 (2014).

[4] E. H. Choi, Vacuum Magazine 2, 9 (2015).

[5] G. L. Geison, Isis 60, 273 (1969).

[6] J. S. Sousa, G. Bauville, B. Lacour, V. Puech, M. Touzeau, and J. L. Ravanat, Appl. Phys. Lett. 97, 141502 (2010).

[7] U. Kogelschatz, Plasma Chem. Plasma Process. 23, 1 (2003).

[8] J. Winter, K. Wende, K. Masur, S. Iseni, M. Dünnbier, M. U. Hammer, H. Tresp, K. D. Weltmann, and S. Reuter, J. Phys. D: Appl. Phys. 46, 295401 (2013).

[9] A. Fridman, A. Chirokov, and A. Gutsol, J. Phys. D: Appl. Phys. 38, R1 (2005).

[10] F. Massines and G. Gouda, J. Phys. D: Appl. Phys. 31, 3411 (1998).

[11] E. H. Choi, H. S. Uhm, and N. K. Kaushik, AAPPS Bull. 31, 10 (2021).

[12] P. Attri, Y. H. Kim, D. H. Park, J. H. Park, Y. J. Hong, H. S. Uhm, K. N. Kim, A. Fridman, and E. H. Choi, Sci. Rep. 5, 9332 (2015).

[13] N. Barekzi and M. Laroussi, IEEE Trans. Plasma Sci. 42, 2738 (2014).

[14] N. Barekzi and M. Laroussi, Plasma Process. Polym. 10, 1039 (2013).

[15] N. Kaushik, N. Uddin, G. B. Sim, Y. J. Hong, K. Y. Baik, C. H. Kim, S. J. Lee, N. K. Kaushik, and E. H. Choi, Sci. Rep. 5, 8587 (2015).

[16] V. Adler, Z. Yin, K. D. Tew, and Z. Ronai, Oncogene 18, 6104 (1999).

[17] J. Benedikt, M. M. Hefny, A. Shaw, B. R. Buckley, F. Iza, S. Schäkermann, and J. E. Bandow, Phys. Chem. Chem. Phys. 20, 12037 (2018). 
[18] S. Mohades, N. Barekzi, H. Razavi, V. Maruthamuthu, and M. Laroussi, Plasma Process. Polym. 13, 1206 (2016).

[19] E. Turrini, A. Stancampiano, E. Simoncelli, R. Laurita, E. Catanzaro, C. Calcabrini, M. Gherardi, V. Colombo, and C. Fimognari, Clin. Plasma Med. 9, 15 (2018).

[20] L. Lin, L. Wang, Y. Liu, C. Xu, Y. Tu, and J. Zhou, Oncol. Rep. 40, 3405 (2018)

[21] N. Kaushik, S. J. Lee, T. G. Choi, K. Y. Baik, H. S. Uhm, C. H. Kim, N. K. Kaushik, and E. H. Choi, Sci. Rep. 5, 8726 (2015).

[22] A. G. Lin, B. Xiang, D. J. Merlino, T. R. Baybutt, J. Sahu, A. Fridman, A. E. Snook, and V. Miller, OncoImmunology 7, e1484978 (2018).

[23] A. Lin et al., Adv. Sci. 6, 1802062 (2019).

[24] B. Haertel, T. V. Woedtke, K. D. Weltmann, and U. Lindequist, Biomol. Ther. 22, 477 (2014).

[25] M. Keidar, R. Walk, A. Shashurin, P. Srinivasan, A. Sandler, S. Dasgupta, R. Ravi, R. G. Preston, and B. Trink, Br. J. Cancer 105, 1295 (2011).

[26] L. Haralambiev et al., Oncol. Lett. 19, 283 (2019).

[27] I. Adamovich et al., J. Phys. D: Appl. Phys. 50, 323001 (2017).

[28] D. Yan et al., ACS Appl. Mater. Interfaces 12, 34548 (2020).

[29] M. Adhikari et al., Cell Commun. Signal. 17, 52 (2019).

[30] G. Chen, Z. Chen, D. Wen, Z. Wang, H. Li, Y. Zeng, G. Dotti, R. E. Wirz, and Z. Gu, Proc. Natl. Acad. Sci. 117, 3687 (2020).

[31] N. K. Kaushik, N. Kaushik, M. Adhikari, B. Ghimire, N. N. Linh, Y. K. Mishra, S. J. Lee, and E. H. Choi, Cancers 11, 842 (2019).

[32] N. K. Kaushik, N. Kaushik, N. N. Linh, B. Ghimire, A. Pengkit, J. Sornsakdanuphap, S. J. Lee, and E. H. Choi, Nanomaterials 9 , 98 (2019).

[33] N. K. Kaushik, N. Kaushik, K. C. Yoo, N. Uddin, J. S. Kim, S. J. Lee, and E. H. Choi, Biomaterials 87, 118 (2016).

[34] N. K. Kaushik, N. Kaushik, R. Wahab, P. Bhartiya, N. N. Linh F. Khan, A. A. AlKhedhairy, and E. H. Choi, Cancers 12, 457 (2020).

[35] G. Isbary, T. Shimizu, Y. F. Li, W. Stolz, H. M. Thomas, G. E. Morfill, and J. L. Zimmermann, Expert Rev. Med. Devices 10, 367 (2014).

[36] N. M. Nasir, B. K. Lee, S. S. Yap, K. L. Thong, and S. L. Yap, Arch. Biochem. Biophys. 605, 76 (2016).

[37] M. Turner, Chapter 2 - Physics of Cold Plasma, Cold Plasma in Food and Agriculture (Academic Press, 2016), pp. 17-51.

[38] T. V. Woedtke, S. Emmert, H. R. Metelmann, S. Rupf, and K. D. Weltmann, Phys. Plasmas 27, 070601 (2020).

[39] S. K. Pankaj and K. M. Keener, Curr. Opin. Food Sci. 16, 49 (2017).

[40] T. Bernhardt, M. L. Semmler, M. Schäfer, S. Bekeschus, S. Emmert, and L. Boeckmann, Oxidative Med. Cell. Longev. 2019, 3873928 (2019)

[41] T. V. Woedtke, A. Schmidt, S. Bekeschus, K. Wende, and K. D. Weltmann, In Vivo 33, 1011 (2019).

[42] M. Laroussi, Front. Phys. 8, 74 (2020).

[43] K. D. Weltmann and T. V. Woedtke, Plasma Phys. Control. Fusion 59, 014031 (2017)

[44] M. G. Kong, G. Kroesen, G. Morfill, T. Nosenko, T. Shimizu, J. V. Dijk, and J. L. Zimmermann, New J. Phys. 11, 115012 (2009).

[45] A. Malyavko, D. Yan, Q. Wang, A. L. Klein, K. C. Patel, J. H. Sherman, and M. Keidar, Mater. Adv. 1, 1494 (2020).

[46] D. Liu, E. J. Szili, and K. Ostrikov, Plasma Process. Polym. 17, 2000097 (2020).

[47] X. Lu, M. Keidar, M. Laroussi, E. H. Choi, E. J. Szili, and K. Ostrikov, Mater. Sci. Eng. R Rep. 138, 36 (2019).

[48] M. Ito, J. S. Oh, T. Ohta, M. Shiratani, and M. Hori, Plasma Process. Polym. 15, 1700073 (2018).

[49] J. S. Song, S. B. Kim, S. Ryu, J. Oh, and D. S. Kim, Front. Plant Sci. 11, 988 (2020).
[50] P. Attri, K. Ishikawa, T. Okumura, K. Koga, and M. Shiratani, Processes 8, 1002 (2020).

[51] B. Adhikari, K. Pangomm, M. Veerana, S. Mitra, and G. Park, Front. Plant Sci. 11, 77 (2020).

[52] P. J. Cullen, J. Lalor, L. Scally, D. Boehm, V. Milosavljević, P. Bourke, and K. Keener, Plasma Process. Polym. 15, 1700085 (2018).

[53] G. Pauzaite, A. Malakauskiene, Z. Nauciene, R. Zukiene, I. Filatova, V. Lyushkevich, I. Azarko, and V. Mildaziene, Plasma Process. Polym. 15, 1700068 (2018).

[54] V. Mildaziene et al., Plasma Process. Polym 15, 1700059 (2018).

[55] C. Szőke, Z. Nagy, K. Gierczik, A. Székely, T. Spitkól, Z. T. Zsuboril, G. Galiba, C. L. Marton, and K. Kutasi, Plasma Process. Polym. 15, 1700138 (2018).

[56] Y. Park, K. S. Oh, J. Oh, D. C. Seok, S. B. Kim, S. J. Yoo, and M. J. Lee, Plasma Process. Polym. 15, 1600056 (2018).

[57] V. Štěpánová, P. Slavíček, J. Kelar, J. Prášil, M. Smékal, M. Stupavská, J. Jurmanová, and M. Černák, Plasma Process. Polym. 15, 1700076 (2018).

[58] C. Smet, M. Baka, A. Dickenson, J. L. Walsh, V. P. Valdramidis, and J. F. V. Impe, Plasma Process. Polym. 15, 1700048 (2018).

[59] B. Adhikari, M. Adhikari, B. Ghimire, G. Park, and E. H. Choi, Sci. Rep. 9, 16080 (2019).

[60] G. Cho, E. H. Choi, and H. S. Uhm, IEEE Trans. Plasma Sci. 41, 1635 (2013).

[61] J. Sornsakdanuphap, P. Suanpoot, Y. J. Hong, B. Ghimire, G. Cho, H. S. Uhm, D. Kim, Y. J. Kim, and E. H. Choi, J. Korean Phys. Soc. 70, 979 (2017)

[62] Y. P. Raizer, Gas Discharge Physics (Springer-Verlag, Berlin, 1991), p52.

[63] A. Fridman, Plasma Chemistry (Cambridge University Press, New York, 2008), p19.

[64] M. Mirzaee, M. S. Simeni, and P. J. Bruggeman, Phys. Plasmas $27,123505(2020)$

[65] M. Laroussi, X. Lu, and M. Keidar, J. Appl. Phys. 122, 020901 (2017).

[66] J. S. Lim, Y. J. Hong, B. Ghimire, J. Choi, S. Mumtaz, and E. H. Choi, Results Phys. 20, 103693 (2021).

[67] F. Yang, Z. Mu, and J. Zhang, Plasma Sci. Technol. 18, 79 (2016).

[68] S. Nunomura, M. Kondo, and H. Akatsuka, Plasma Sources Sci. Technol. 15, 783 (2006)

[69] P. Svarnas, Plasma Sci. Technol. 15, 891 (2013).

[70] S. Park, W. Choe, H. Lee, J. Y. Park, J. Kim, S. Y. Moon, and U. Cvelbar, Nature 592, 49 (2021).

[71] P. Shaw, N. Kumar, H. S. Kwak, J. H. Park, H. S. Uhm, A. Bogaerts, E. H. Choi, and P. Attri, Sci. Rep. 8, 11268 (2018).

[72] S. Jung, H. J. Kim, S. Park, H. I. Yong, J. H. Choe, H. J. Jeon, W. Choe, and C. Jo, Meat Sci. 108, 132 (2015).

[73] J. Šimečková, F. Krčma, D. Klofáč, L. Dostál, and Z. Kozáková, Water 12, 2357 (2020).

[74] U. Schnabel et al., Foods 8, 55 (2019).

[75] H. Tanaka, M. Mizuno, K. Ishikawa, K. Nakamura, H. Kajiyama, H. Kano, F. Kikkawa, and M. Hori, Plasma Med. 1, 265 (2011).

[76] A. Azzariti et al., Sci. Rep. 9, 4099 (2019).

[77] N. Yoshikawa et al., Sci. Rep. 10, 1614 (2020).

[78] P. Attri, J. H. Park, A. Ali, and E. H. Choi, Anti-Cancer Agents Med. Chem. 18, 805 (2018).

[79] 21CFR801.415 - Maximum acceptable level of ozone: KS C9314: Standards of interior air cleaner (SPS-KACA 0020132): Criterion for chemical substance and physical factor exposue (Ministry of Employment and Labor Notice \#2020-48).

[80] Evaluation guideline for plasma wound treatment (Ministry of Food and Drug and Safety, Korean Government, 2016.12)

[81] J. S. Kwon, S. H. Choi, E. H. Choi, K. M. Kim, and P. K. Chu, Int. J. Mol. Sci. 21, 6085 (2020) 
[82] S. Mitra, P. Bhartiya, N. Kaushik, L. N. Nguyen, R. Wahab, S. Bekeschus, E. H. Choi, and N. K. Kaushik, Appl. Sci. 10, 8395 (2020).

[83] H. Noh, J. E. Kim, J. Y. Kim, S. H. Kim, I. Han, J. S. Lim, S. H. Ki, E. H. Choi, and G. J. Lee, Appl. Sci. 9, 3921 (2019).

[84] M. Adhikari, B. Adhikari, B. Ghimire, S. Baboota, and E. H. Choi, Int. J. Mol. Sci. 21, 1939 (2020).

[85] E. H. Choi, appeared in Book Chapter 3, Edited by M. Keidar, Plasma Cancer Therapy (Springer, 2020).

[86] B. Ghimire, J. Sornsakdanuphap, Y. J. Hong, H. S. Uhm, K. D. Weltmann, and E. H. Choi, Phys. Plasmas 24, 073502 (2017).

[87] B. Ghimire, P. Lamichhane, J. S. Lim, B. Min, R. Paneru, K. D. Weltmann, and E. H. Choi, Appl. Phys. Lett. 113, 194101 (2018).

[88] J. S. Lim, R. H. Kim, Y. J. Hong, P. Lamichhane, B. C. Adhikari, J. Choi, and E. H. Choi, Results Phys. 19, 103569 (2020).

[89] B. Ghimire et al., Appl. Phys. Lett. 114, 093701 (2019).

[90] J. Duan, X. Lu, and G. He, Phys. Plasmas 24, 073506 (2017).

[91] Test Result for COVID19 (SARS-CoV-2 virus), Q2017071, Issued from Korea University (2020).

[92] P. Lamichhane, B. C. Adhikari, L. N. Nguyen, R. Paneru, B. Ghimire, S. Mumtaz, J. S. Lim, Y. J. Hong, and E. H. Choi, Plasma Sources Sci. Technol. 29, 045026 (2020).

[93] D. K. Yadav, M. Adhikari, S. Kumar, B. Ghimire, I. Han, M. H. Kim, and E. H. Choi, Sci. Rep. 10, 3396 (2020).

[94] W. Tian and M. J. Kushner, J. Phys. D: Appl. Phys. 48, 494002 (2015).

[95] A. Starikovskiy, Y. Yang, Y. I. Cho, and A. Fridman, Plasma Sources Sci. Technol. 20, 024003 (2011).

[96] B. Sun, S. Kunitomo, and C. Igarashi, J. Phys. D: Appl. Phys. 39, 3814 (2006)

[97] M. Sahni, and B. Locke, Plasma Process. Polym. 3, 342 (2006).

[98] P. Lukes, A. T. Appleton, and B. R. Locke, IEEE Trans. Ind. Appl. 40, 60 (2004).

[99] P. Lukes, M. Clupek, V. Babicky, and P. Sunka, Plasma Sources Sci. Technol. 17, 024012 (2008).

[100] H. Jablonowski, R. Bussiahn, M. U. Hammer, K. D. Weltmann, T. V. Woedtke, and S. Reuter, Phys. Plasmas 22, 122008 (2015).

[101] P. J. Bruggeman et al., Plasma Sources Sci. Technol. 25, 053002 (2016).

[102] T. He, D. Liu, Z. Liu, S. Wang, Z. Liu, M. Rong, and M. G. Kong, J. Phys. D: Appl. Phys. 52, 045204 (2019).

[103] B. Halliwell and J. M. C. Gutteridge, Free Radicals in Biology and Medicine (Oxford Univeristy Press, 2015).

[104] Y. J. Hong, C. J. Nam, K. B. Song, G. S. Cho, H. S. Uhm, D. I. Choi, and E. H. Choi, J. Instrum. 7, C03046 (2012).

[105] Y. H. Kim, Y. J. Hong, K. Y. Baik, G. C. Kwon, J. J. Choi, G. S. Cho, H. S. Uhm, D. Y. Kim, and E. H. Choi, Plasma Chem. Plasma Process. 34, 457 (2014).

[106] H. P. Dorn, R. Neuroth, and A. Hofzumahaus, J. Geophys. Res. Atmos. 100, 7397 (1995).

[107] N. Srivastava and C. Wang, J. Appl. Phys. 110, 053304 (2011).

[108] M. Baeva, K. Rackow, M. M. Becker, J. Ehlbeck, and D. Loffhagen, 30th ICPIG (Belfast, Northern Ireland, 2011), Topic C9.

[109] A. Sarani, A. Y. Nikiforov, and C. Leys, Phys. Plasmas 17 063504 (2010).

[110] B. Benstaali, P. Boubert, B. G. Cheron, A. Addou, and J. L. Brisset, Plasma Chem. Plasma Process. 22, 553 (2002).

[111] N. C. Roy, M. G. Hafez, and M. R. Talukder, Phys. Plasmas 23, 83502 (2016)

[112] P. Bruggeman and D. C. Schram, Plasma Sources Sci. Technol 19, 045025 (2010).

[113] D. X. Liu, P. Bruggeman, F. Iza, M. Z. Rong, and M. G. Kong, Plasma Sources Sci. Technol. 19, 25018 (2010).

[114] A. S. Bleker, J. Winter, A. Bösel, S. Reuter, and K. D. Weltmann, Plasma Sources Sci. Technol. 25, 015005 (2015).
[115] Y. J. Hong, J. Lim, J. S. Choi, K. D. Weltmann, and E. H. Choi, Plasma Process Polym. 18, e2000168 (2021).

[116] G. Berden and R. Engeln, Cavity Ring-Down Spectroscopy Techniques and Applications (Wiley-Blackwell, 2009).

[117] S. Kassi, K. Didriche, C. Lauzin, X. de Ghellinck d'Elseghem Vaernewijck, A. Rizopoulos, and M. Herman, Spectrochim. Acta A Mol. Biomol. Spectrosc. 75, 142 (2010).

[118] Z. Du, S. Zhang, J. Li, N. Gao, and K. Tong, Appl. Sci. 9, 338 (2019).

[119] T. E. L. Smith, M. J. Wooster, M. Tattaris, and D. W. T. Griffith Atmos. Meas. Tech. 4, 97 (2011).

[120] S. Iseni, S. Reuter, and K. D. Weltmann, J. Phys. D: Appl. Phys. 47, 075203 (2014)

[121] J. P. Burrows, A. Dehn, B. Deters, S. Himmelmann, A. Richter, S. Voigt, and J. Orphal, J. Quant. Spectrosc. Radiat. Transfer 60, 1025 (1998)

[122] J. Wojtas, Z. Bielecki, T. Stacewicz, J. Mikolajczyk, R. Medrzycki, and B. Rutecka, Acta Phys. Pol. A 120, 794 (2011).

[123] S. Park, H. Kim, H. W. Ji, H. W. Kim, S. H. Yun, E. H. Choi, and S. J. Kim, Cancers 11, 2011 (2019)

[124] L. Lin, L. Wang, Y. Liu, C. Xu, Y. Tu, and J. Zhou, Oncol. Rep. 40, 3405 (2018).

[125] J. Köritzer et al., PLoS One 8, e64498 (2013).

[126] L. N. Nguyen, N. Kaushik, P. Bhartiya, S. K. Gurmessa, H. J. Kim, L. Q. Nguyen, N.K. Kaushik, and E. H. Choi, J. Ind. Eng. Chem. 100, 99 (2021).

[127] N. K. Kaushik, N. Kaushik, B. Min, K. H. Choi, Y. J. Hong, V. Miller, A. Fridman, and E. H. Choi, J. Phys. D: Appl. Phys. 49, 084001 (2016).

[128] G. C. Kim, G. J. Kim, S. R. Park, S. M. Jeon, H. J. Seo, F. Iza, and J. K. Lee, J. Phys. D: Appl. Phys. 42, 032005 (2008).

[129] B. B. Choi, Y. S. Choi, H. J. Lee, J. K. Lee, U. K. Kim, and G. C. Kim, J. Therm. Sci. Technol. 7, 399 (2012).

[130] L. N. Nguyen et al., Green Chem. 22, 6588 (2020).

[131] K. H. Choi, E. W. Choi, J. Min, H. Son, H. S. Uhm, E. H. Choi, B. J. Park, and J. S. Jung, IEEE Trans. Magn. 50, 1 (2014).

[132] B. J. Park et al., J. Biomed. Nanotechnol. 11, 226 (2015).

[133] D. Yan, J. H. Sherman, and M. Keidar, Oncotarget 8, 15977 (2017).

[134] J. Hou, J. Ma, K. N. Yu, W. Li, C. Cheng, L. Bao, and W. Han, BMC Genomics 16, 435 (2015).

[135] H. Tanaka et al., Sci. Rep. 9, 13657 (2019).

[136] F. R. Balkwill, M. Capasso, and T. Hagemann, J. Cell Sci. 125, 5591 (2012).

[137] A. P. Maldonado, C. Bengtson, J. Razzokov, E. Smits, and A. Bogaerts, Cancers 11, 1920 (2019).

[138] M. Vandamme, E. Robert, S. Pesnel, E. Barbosa, S. Dozias, J. Sobilo, S. Lerondel, A. L. Pape, and J. M. Pouvesle, Plasma Process. Polym. 7, 264 (2010).

[139] L. I. Partecke et al., BMC Cancer 12, 473 (2012).

[140] T. V. Woedtke, H. R. Metelmann, and K. D. Weltmann, Contrib. to Plasma Phys. 54, 104 (2014).

[141] H. Kajiyama, K. Nakamura, F. Utsumi, H. Tanaka, M. Hori, and F. Kikkawa, Jpn. J. Appl. Phys.53, 05FA05 (2014).

[142] E. Freund, K. R. Liedtke, J. V. D. Linde, H. R. Metelmann, C. D. Heidecke, L. I. Partecke, and S. Bekeschus, Sci. Rep. 9, 634 (2019).

[143] F. Utsumi et al., PLoS ONE 8, e81576 (2013).

[144] N. Jha, J. J. Ryu, E. H. Choi, and N. K. Kaushik, Oxid. Med. Cell. Longev. 2017, 7542540 (2017).

[145] A. D. Morris, G. B. McCombs, T. Akan, W. Hynes, M. Laroussi, and S. L. Tolle, J. Dent. Hyg. 83, 55 (2009).

[146] M. Laroussi, D. A. Mendis, and M. Rosenberg, New J. Phys. 5, 41 (2003).

[147] P. E. Petersen, Community Dent. Oral Epidemiol. 31, 3 (2003).

[148] Y. Li, J. Pan, G. Ye, Q. Zhang, J. Wang, J. Zhang, and J. Fang, 
Eur. J. Oral Sci. 125, 463 (2017).

[149] Kang, Z. Q. The bleaching efficiency and bio-safety assessment of Plasma Activated Water by low conc. $\mathrm{H}_{2} \mathrm{O}_{2}$. Global Thesis. (2016).

[150] Y. C. Cheng, C. H. Wu, C. T. Liu, C. Y. Lin, H. P. Chiang, T. W. Chen, C. Y. Chen, and J. S. Wu, Plasma Process. Polym. 14, 1600235 (2017).

[151] N. K. Kaushik et al., Biol. Chem. 400, 39 (2018).

[152] R. Ranjan, P. V. Krishnamraju, T. Shankar, and S. Gowd, J. Int. Soc. Prev. Community Dent. 7, 71 (2017).

[153] M. Gherardi, R. Tonini, and V. Colombo, Trends Biotechnol. 36, 583 (2018).

[154] S. J. Sung, J. B. Huh, M. J. Yun, B. M. W. Chang, C. M. Jeong, and Y. C. Jeon, J Adv Prosthodont 5, 2 (2013).

[155] N. Jha, J. J. Ryu, R. Wahab, A. A. A. Khedhairy, E. H. Choi, and N. K. Kaushik, Oncotarget 8, 20496 (2017).

[156] J. S. Kwon, Y. H. Kim, E. H. Choi, C. K. Kim, K. N. Kim, and K. M. Kim, Clin. Oral Investig. 20, 1801 (2016).

[157] Y. Zhang, Q. Yu, and Y. Wang, J. Dent. 42, 1033 (2014)

[158] A. P. Ayres, P. H. Freitas, J. D. Munck, A. Vananroye, C. Clasen, C. T. D. S. Dias, M. Giannini, and B. V. Meerbeek, Oper. Dent. 43, E288 (2018)

[159] W. S. Jeong, J. S. Kwon, E. H. Choi, and K. M. Kim, Sci. Rep. 8 , 15963 (2018).

[160] T. Lu, Y. Qiao, and X. Liu, Interface Focus 2, 325 (2012).

[161] G. C. Yeo, M. Santos, A. Kondyurin, J. Liskova, A. S. Weiss, and M. M. M. Bilek, ACS Biomater. Sci. Eng. 2, 662 (2016).

[162] A. Nasir and G. C. Anollés, Sci. Adv. 1, e1500527 (2015).

[163] S. W. Wilhelm and C. A. Suttle, BioScience 49, 781 (1999).

[164] L. Guo, R. Xu, L. Gou, Z. Liu, Y. Zhao, D. Liu, L. Zhang, H. Chen, and M. G. Kong, Appl. Environ. Microbiol. 84, e00726 (2018).

[165] A. Filipić, I. G. Aguirre, G. Primc, M. Mozetič, and D. Dobnik, Trends Biotechnol. 38, 1278 (2020).

[166] D. Dobrynin, G. Fridman, G. Friedman, and A. Fridman, New J. Phys. 11, 115020 (2009).
[167] C. Tendero, C. Tixier, P. Tristant, J. Desmaison, and P. Leprince, Spectrochim. Acta B: At. Spectrosc. 61, 2 (2006).

[168] M. Laroussi, IEEE Trans. Plasma Sci., 24, 1188 (1996).

[169] S. Bekeschus, A. Kramer, E. Suffredini, T. V. Woedtke, and V. Colombo, IEEE Trans. Radiat. Plasma Med. Sci. 4, 391 (2020).

[170] Z. Chen, G. Gustavo Jr, V. Arumugaswami, and R. E. Wirz, Phys. Fluids 32, 111702 (2020).

[171] R. Kar et al., Trans. Indian Natl. Acad. Eng. 5, 327 (2020).

[172] A. Bisag et al., Plasma Process Polym. 17, 2000154 (2020).

[173] T. Xia, A. Kleinheksel, E. M. Lee, Z. Qiao, K. R. Wigginton, and H. L. Clack, J. Phys. D: Appl. Phys. 52, 255201 (2019).

[174] L. Guo et al., Chem. Eng. J. 421, 127742 (2020).

[175] P. Attri, K. Koga, and M. Shiratani, Appl. Phys. Express 14, $027002(2021)$

[176] Z. Chen, G. Garcia Jr., V. Arumugaswami, and R. E. Wirz, Phys. Fluids 32, 111702 (2020)

[177] M. Weiss, G. Daeschlein, A. Kramer, M. Burchardt, S. Brucker, D. Wallwiener, and M. B. Stope, J. Med. Virol. 89, 952 (2017).

[178] N. H. Kim, Y. K. Hong, and S. H. Lee, J. Pain Res. 11, 2229 (2018).

[179] Y. Hong, S. Yoo, N. H. Kim, Y. Kim, S. Sohn, S. Y. Yoon, and S Lee, J. Neurol. Surg. A: Cent. Eur. Neurosurg. 79, 231 (2018).

[180] S. Y. Yoon, G. H. Kim, Y. Kim, N. H. Kim, S. Lee, C. Kawai, and Y. Hong, J. Neurol. Surg. A: Cent. Eur. Neurosurg. 80, 034 (2019).

[181] P. Attri, N. K. Kaushik, N. Kaushik, D. Hammerschmid, A. P. Maldonado, J. D. Backer, M. Shiratani, E. H. Choi, and A. Bogaerts, Int. J. Biol. Macromol. 182, 1724 (2021).

[182] A. Barjasteh, Z. Dehghani, P. Lamichhane, N. Kaushik, E. H. Choi, and N. K. Kaushik, Appl. Sci. 11, 3372 (2021).

[183] N. K. Kaushik, S. Bekeschus, H. Tanaka, A. Lin, and E. H. Choi, Appl. Sci. 11, 4584 (2021).

[184] P. Lamichhane, M. Veerana, J. S. Lim, S. Mumtaz, B. Shrestha, N. K. Kaushik, G. Park, and E. H. Choi. Int. J. Mol. Sci. 22, 5360 (2021). 